\title{
Structural properties of isolated early-type galaxies. The dependence of scaling relations on environment ${ }^{\star}$
}

\author{
A. Nigoche-Netro ${ }^{1}$, M. Moles ${ }^{1}$, A. Ruelas-Mayorga ${ }^{2}$, A. Franco-Balderas ${ }^{2}$, and P. Kjærgaard ${ }^{3}$ \\ 1 Instituto de Astrofísica de Andalucía (IAA), Camino bajo de Huetor 24, 18008 Granada, Spain \\ e-mail: alberto.n@imaff.cfmac.csic.es; moles@iaa.es \\ ${ }^{2}$ Instituto de Astronomía, Universidad Nacional Autónoma de México. Apartado Postal 70-264, México D.F., C.P. 04510, México \\ e-mail: rarm@astrocu.unam.mx \\ 3 Copenhagen University Observatory, The Niels Bohr Institute For Astronomy, Physics and Geophysics, Juliane Maries Vej 30, \\ 2100 Copenhagen, Denmark e-mail: per@astro.ku.dk
}

Received 14 September 2006 / Accepted 18 May 2007

\section{ABSTRACT}

\begin{abstract}
Aims. In order to investigate whether gravitational interactions between galaxies are able to modify their structural properties, we determine the Kormendy relation (KR) for a sample of isolated early-type galaxies (ETG) for which we have measured several photometric parameters. This relation is compared with that obtained for a sample of ETG of the Coma Cluster, and with that obtained for a sample of perturbed S0 galaxies contained within high-density environments.

Methods. Using very strict physical isolation criteria we selected a sample of $82 \mathrm{E}$ and S0 galaxies from the CfA catalogue, in the redshift range $5000 \mathrm{~km} \mathrm{~s}^{-1} \leq c z<10000 \mathrm{~km} \mathrm{~s}^{-1}$. We obtained the photometric parameters, $\left(r_{\mathrm{e}},\left\langle S B_{\mathrm{e}}\right\rangle\right)$ and total magnitude for 21 galaxies in the $B$ filter and for 23 in the Gunn $r$ filter.

Results. The main result we obtained is that the KR is the same for isolated galaxies and for galaxies in high-density environments or in interaction. In addition we found evidence that the intrinsic dispersion of the KR depends on the magnitude range within which the galaxies are contained (larger dispersion for larger magnitude range), and not on the degree of gravitational interaction to which these galaxies are subjected.
\end{abstract}

Key words. galaxies: elliptical and lenticular, $\mathrm{cD}$ - galaxies: fundamental parameters - galaxies: interactions

\section{Introduction}

It is well known that some galactic properties such as morphologic type or star formation rate are correlated with the environment in which a galaxy is found. Dressler (1980) demonstrated that red ETG are found more frequently in clusters or groups rather than in the field. Further studies confirmed these results and demonstrated that other properties such as the mass and the luminosity also depend on the environment (Whitmore et al. 1993; Balogh 1997; Hashimoto et al. 1998; Poggianti et al. 1999; Couch et al. 2001; Gómez et al. 2003; Kauffmann et al. 2004; Tanaka et al. 2004; Blanton et al. 2003; Berlind et al. 2005). Similarly, Varela et al. (2004) concluded that isolated disk galaxies are less luminous, bluer and smaller than galaxies of the same types in more dense environments.

The question of the influence of the environment on galactic properties has also been addressed through the analysis of scaling relations. de Carvalho \& Djorgovski (1992) found that field ETG follow the same structural relations as galaxies in clusters but with a larger intrinsic scatter in their properties. More recently, Bernardi et al. (2003), analysed the Fundamental Plane (FP) relation for a sample of $\sim 9000$ ETG and contrary to the claims by de Carvalho and Djorgovski, found a lower intrinsic dispersion for field galaxies as opposed to those in denser environments. This is in agreement with Márquez \& Moles (1996, 1999), who found that the relation between the

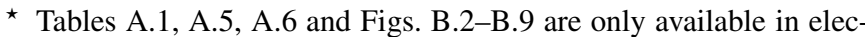
tronic form at http://www . aanda.org bulge parameters of isolated spirals presents a lower scatter than for non-isolated systems. Pursuing the same line of research, but using the line-strength index- $\sigma_{0}$ relation, Denicoló et al. (2005) found that it does not seem to change with the environmental density even if the scatter of the relation appears larger for isolated, group and field galaxies than for galaxies in clusters. Bernardi et al. (2003) assert that the dispersion around the structural relations might represent evidence that ETG contained in dense regions are slightly less luminous than ETG found in less dense regions, and that, as a consequence, ETG contained within dense regions must have been formed at high redshifts.

The previously mentioned results claim that differences (in one sense or another) are dependent on the environment. However, de la Rosa et al. (2001) concluded that elliptical galaxies in very loose groups or in the field, show the same FP relation as galaxies in more dense environments, such as those in compact groups and clusters (see also Jørgensen et al. 1993). Similarly, Treu et al. (2001) found that the intercept and scatter of the FP for field ETG and for cluster galaxies are mutually consistent, whithin the observational errors. These conclusions are strengthened by the work of Evstigneeva et al. (2002) who, using numerical experiments and observational data, have shown that the global parameters for ETG are stable to gravitational perturbations (see also Kjærgaard et al. 1993). FP parameters changed during close encounters but within a very short time-interval $\left(\sim 10^{7}-10^{8}\right.$ years $)$ and the amplitude of the changes is comparable to the dispersion in the values of the observed 
structural relations. Simulations by González-García \& van Albada (2003) of the effects of merging on the FP relation, show that the merger remnants of elliptical galaxies lie very close to the FP of the progenitors. Thus, merging would not change appreciably the FP relation since the scatter introduced in the relation is smaller than that due to the projection effects. Finally, Reda et al. $(2004,2005)$ find that isolated galaxies follow the same photometric relations as galaxies in high density environments and that most isolated galaxies follow the same FP tilt and scatter as galaxies in high-density environments. However, a few galaxies deviate notably from the plane in the sense of having smaller $\mathrm{M} / \mathrm{L}$ ratios. This latter fact is interpreted by Reda et al. (2005) by invoking the presence of younger stellar populations, which are presumably induced by a gaseous merger.

The various studies mentioned above, deal with different aspects of structural relations for ETG in different environments. Discrepancies between them are notorious. Some might be due to the selection criteria used to define field and/or isolated galaxies. In fact, among what is often presented as isolated galaxies, it is rather frequent to find a significant fraction of galaxies showing signatures of recent perturbations, as discussed by Márquez \& Moles (1996), Varela et al. (2004) or in Reda et al. (2004). In fact, we still lack a sample of truly isolated or even mildly interacting ETG to gauge the effects of interaction. Indeed, if the properties depend on the local density, then this is a very relevant aspect.

In this paper we present a sample of well defined isolated ETG to address their structural properties and the scaling relations they define. Indeed, defining such a sample is not straightforward for two main reasons: i) the relatively low proportion of this type of object in low density environments; and ii) the difficulty in defining a physically sound and operational isolation criteria. Based on previous work by Varela et al (2004), we present here a sample of 82 isolated ETG using very strict isolation criteria. Part of the sample was observed to obtain the photometric parameters to build the KR and compare it with that obtained for samples in dense environments.

In Sect. 2 we describe the selection process of our isolated ETG and their properties. In Sect. 3 we present the observations, the reduction method and the results. In Sect. 4 we describe the compilation and properties of two galaxy samples from the literature; a high density ETG sample in the Coma cluster and a perturbed S0-galaxy sample. In Sect. 5 we analyse the KR for the samples under different density conditions. Finally in Sect. 6 we present our results and conclusions. In Appendices A and B we present the data for the galaxies compiled in this paper. Throughout this paper we use a Hubble constant of $70 \mathrm{~km} \mathrm{~s}^{-1} \mathrm{Mpc}^{-1}$.

\section{Sample selection}

The sample of E and S0 galaxies for this study was extracted from the CfA Galaxy Catalogue (Huchra et al. 2000). The selection criteria we used are those defined in Varela et al. (2004) (see also Márquez \& Moles 1996). The process was as follows:

- Selection from the CfA of the galaxies with morphological type coded as $-7 \leq t \leq 0$.

- Selection of the redshift range, $0.017 \leq z \leq 0.033$. Given that the determination of the distances to the galaxies relies entirely on the redshift, we chose the lower limit to keep the influence of the possible streaming motions on the measured $z$ below $10 \%$. The upper limit was imposed to obtain a bright enough sample consisting of galaxies that are not too small in size.

- The galactic latitude was chosen $|b| \geq 40$, to minimise the galactic extinction.

- For practical reasons the declination was chosen to be $-30 \leq$ $\delta \leq 30$.

The resulting sample was inspected for isolation. Following Márquez \& Moles (1996), galaxies with companions at a projected distance greater than $0.5 \mathrm{Mpc}$ were considered as isolated. When redshift information on the possible companion was also available, the galaxy was considered isolated when the radial velocity difference was bigger than $500 \mathrm{~km} \mathrm{~s}^{-1}$. Otherwise, we adopted the criterion given by Varela et al. (2004), i.e., $f \leq-4.5$, where $f$ is the logarithmic ratio between external and internal forces for a given galaxy in a given environment. As explained in Varela et al, the actual limit was imposed after considering the dynamical situation of galaxies in environments such as the Coma cluster. These limits ensure that selected galaxies were not affected by external forces in their evolution in the last $2 \times 10^{9}$ years at least. We also considered as isolated those galaxies with possible companions, that, placed at the distance of the galaxy, would have very small sizes $(r<2 \mathrm{kpc})$ or very low luminosities $(M>-12)$.

The application of the criteria discussed above to the CfA catalogue, resulted in a sample of $100 \mathrm{E}$ and S0 galaxies. Lastly, we made a visual inspection of these galaxies on images from the Digitized Sky Survey (DSS) and also carried out an exhaustive literature search for information which might indicate possible interactions or associations with clusters or groups. We found 4 galaxies with indications of interaction (such as distorted nucleii, dust lanes or shells) and 17 galaxies with indications of association with groups. For 3 galaxies (PGC 5381, PGC 5655, and PGC 7658) the redshift difference indicates that their association with a group results from a mere projection effect, so the final sample includes $82 \mathrm{E}$ and $\mathrm{S} 0$ galaxies satisfying the isolation criteria. We emphasise that the application of the isolation limit $f \leq-4.5$ ensures that the evolution in terms of structure and stellar content of these galaxies, has been dominated by internal forces for most of their life (Varela et al. 2004). This result was confirmed by Galletta et al. (2006), who argued that the sample selected by Varela et al. is similar to galaxies observed at redshift $z=1.4$, as if they had stopped accreting mass at that epoch. Indeed, this sample of 82 ETG contains only truly isolated galaxies in the sense defined above, but it is by no means complete, since all galaxies with unknown redshift or with possible companions under the $f$-criterion, but with unknown redshift, were discarded.

\subsection{Properties of the isolated early-type galaxy sample}

The sample of isolated E and S0 galaxies is constituted by $61 \mathrm{~S} 0$, 17 normal and dwarf ellipticals, 2 compact ellipticals and 2 nonclassified ellipticals after the CfA denominations. The apparent $\mathrm{B}$ magnitudes given by the CfA are contained within the interval 13-17 mag, even if most galaxies are in a narrower range, 14-16 mag. There are only 5 galaxies fainter than 16 mag and 4 brighter than 14 mag, as can be seen in Fig. 1.

The heliocentric velocity distribution ( $c z$ ) (Fig. 1) shows a maximum around $5500 \mathrm{~km} \mathrm{~s}^{-1}$ and diminishes gradually towards higher velocities. Only 20 galaxies have $c z>$ $8000 \mathrm{~km} \mathrm{~s}^{-1}$. The ratio of elliptical to $\mathrm{E}+\mathrm{S} 0$ galaxies in our sample is $\mathrm{E} /(\mathrm{E}+\mathrm{S} 0) \sim 25 \%$. This ratio is different to that found for field galaxies $(\mathrm{E} /(\mathrm{E}+\mathrm{S} 0) \sim 33 \%)$ or for rich clusters 

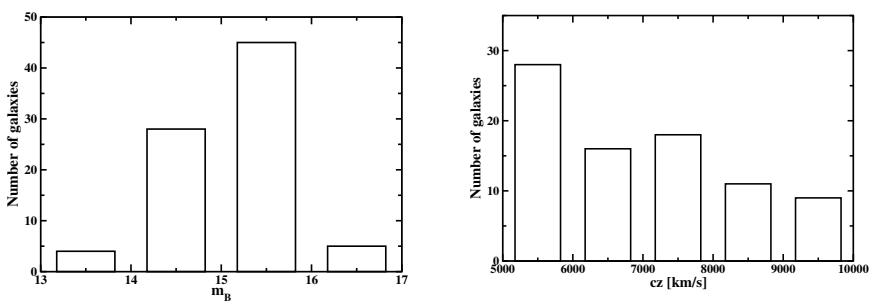

Fig. 1. Magnitude distribution (left) and velocity distribution (right) for the sample of isolated $\mathrm{E}$ and $\mathrm{S} 0$ galaxies.

(E/(E+S0) 43\%) (Bahcall 1977; Oemler 1974; Dressler 1980; Dekel \& Ostriker 1999). We note that Varela et al. (2004) also found a type-effect for disk galaxies, in the sense that isolated galaxies tend to have a later morphological type than nonisolated galaxies. The fact that galaxies appear to have earliertypes in dense environments might be a general property of galaxies. In the case of other samples of E and S0 isolated galaxies from the literature (Reduzzi et al. 1996; Colbert et al. 2001; Aars et al. 2001; Kuntschner et al. 2002; Smith et al. 2003; Stocke et al. 2004; Reda et al. 2004), the E/(E+S0) ratios also differ from those found in this paper. These differences might also be due to intrinsic properties. This is based on recent evidence (Reda et al. 2004) that a large fraction of galaxies in these samples of "isolated" galaxies have faint neighbours in their surroundings, have similar surroundings to the poor groups or have had mergers or accretion in the recent past. Another important question regarding the difference in morphological types in our sample and those in the literature is that the selection criteria used are not the same, i.e. in this paper we consider that a galaxy is an ETG if its morphological type is in the range $-7 \leq t \leq 0$, however, in some of the papers mentioned, such as Kuntschner et al. (2002) and Reda et al. (2004) they consider the early-type defining range as $-7 \leq t \leq-3$ or in the case of Smith et al. (2003) they take the range to be $-7 \leq t \leq-4$.

In Table A.1 (Appendix A) we present the catalogue of the isolated $\mathrm{E}$ and $\mathrm{S} 0$ galaxies including basic data from LEDA such as magnitudes and heliocentric velocities, as well as the morphological information from the CfA catalogue.

\section{Photometric observations and data reduction}

Since existing photometric information for our sample of isolated galaxies is very scarce (only 7 galaxies have surface photometry information) and heterogeneous (different filters and parameter correction methods), we obtained surface photometry information for part of the sample.

The photometric data were obtained during two observing seasons (December 2000 and August 2001) with the Danish $1.5 \mathrm{~m}$ telescope at La Silla (Chile), using the DFOSC instrument, in the $B$ and Gunn $r$ bands. The detector was a $2048 \times 2048$ CCD, with a pixel size of $15 \mu \mathrm{m}$. The corresponding field of view is $13.3^{\prime} \times 13.3^{\prime}$. With a total exposure time per galaxy of about 3000 seconds, a signal to noise ratio $(S / N)$ of $\sim 2$ per pixel for the isophote $S B_{B}=26 \mathrm{mag} \operatorname{arcsec}^{-2}$ and $S / N \sim 2$ per pixel for the isophote $S B_{\text {Gunn } r}=25 \mathrm{mag} \operatorname{arcsec}^{-2}$ was achieved. A minimum of 4 standard stars per night were observed in both filters to correct for atmospheric extinction and to calibrate the flux. The typical seeing was about $1.3 \mathrm{arcsec}$, ranging from 1.0 arcsec to 1.6 arcsec. We could obtain images for 21 galaxies in the Johnson $B$ filter and for 23 galaxies in the Gunn $r$ filter. In Table A.2 (Appendix A) we show the exposure times as well as the seeing data for each of the observed galaxies.
Table 1. Atmospheric extinction coefficients.

\begin{tabular}{ccc}
\hline \hline & Season 1 & \\
Night & $k_{\mathrm{B}}[\mathrm{mag} /$ airmass $]$ & $k_{\text {Gunn } r}[\mathrm{mag} /$ airmass $]$ \\
\hline 27 & $0.215 \pm 0.024$ & $0.0548 \pm 0.010$ \\
28 & $0.220 \pm 0.022$ & $0.0548 \pm 0.009$ \\
29 & $0.217 \pm 0.019$ & $0.0536 \pm 0.007$ \\
30 & $0.224 \pm 0.022$ & $0.0546 \pm 0.011$ \\
31 & $0.212 \pm 0.018$ & $0.0499 \pm 0.009$ \\
01 & $0.216 \pm 0.019$ & $0.0553 \pm 0.012$ \\
02 & $0.241 \pm 0.023$ & $0.0553 \pm 0.008$ \\
03 & $0.233 \pm 0.025$ & $0.0527 \pm 0.009$ \\
\hline & Season 2 & \\
20 & $0.216 \pm 0.016$ & $0.0472 \pm 0.008$ \\
21 & $0.249 \pm 0.011$ & $0.0472 \pm 0.008$ \\
22 & $0.251 \pm 0.027$ & $0.0778 \pm 0.019$ \\
23 & $0.241 \pm 0.022$ & $0.0553 \pm 0.011$ \\
\hline
\end{tabular}

The reduction process was standard, using IRAF ${ }^{1}$ tasks. A somewhat delicate step was the illumination correction in the Gunn $r$ filter, as the images presented pronounced illumination gradients. The reason for this gradient is on one hand the difference in colour between the night-sky and the dusk-sky, since it is during dusk that the flat field correcting images are taken. Moreover, this problem might have been increased due to a red leak in the Gunn $r$ filter (Stetson \& Harris 1988). The illumination correction image was obtained from a combination of deep sky images which contained very few objects. It was smoothed using task MKSKYCOR. Once the correction had been applied, the brightness gradient turned out to be smaller than $1 \%$. Table 1 shows the coefficients of atmospheric extinction for each night for both observing seasons.

The calibration coefficients were obtained following the standard procedure using standard stars from the Thuan-Gunn system (Jørgensen et al. 1996). The transformation equations for both observing runs are as follows:

Season 1.

$(B-G r)_{\mathrm{std}}=0.123+0.957(B-G r)_{\mathrm{inst}}$,

$\sigma_{1}=0.008$

Season 2.

$(B-G r)_{\mathrm{std}}=0.172+0.846(B-G r)_{\mathrm{inst}}$,

$\sigma_{2}=0.107$

Where the subindices std and inst refer to the Standard system and the instrumental system respectively. $\sigma_{1}$ and $\sigma_{2}$ represent the data dispersion around the regression line for Seasons 1 and 2 , respectively.

\subsection{Surface photometry}

Surface photometry was obtained using the task ellipse from IRAF. Since our observed sample contains ETG with absolute magnitude $M_{B} \lesssim-19$ their surface brightness profiles can be well fit by a de Vaucouleurs law (Prugniel \& Siemens 1997). The task $n$ fit $1 d$ was used to fit the data for each of the galaxies,

1 The IRAF package is written and supported by the IRAF programming group at the National Optical Astronomy Observatories (NOAO) in Tucson, Arizona. NOAO is operated by the Association of Universities for Research in Astronomy (AURA), Inc. under cooperative agreement with the National Science Foundation (NSF). 

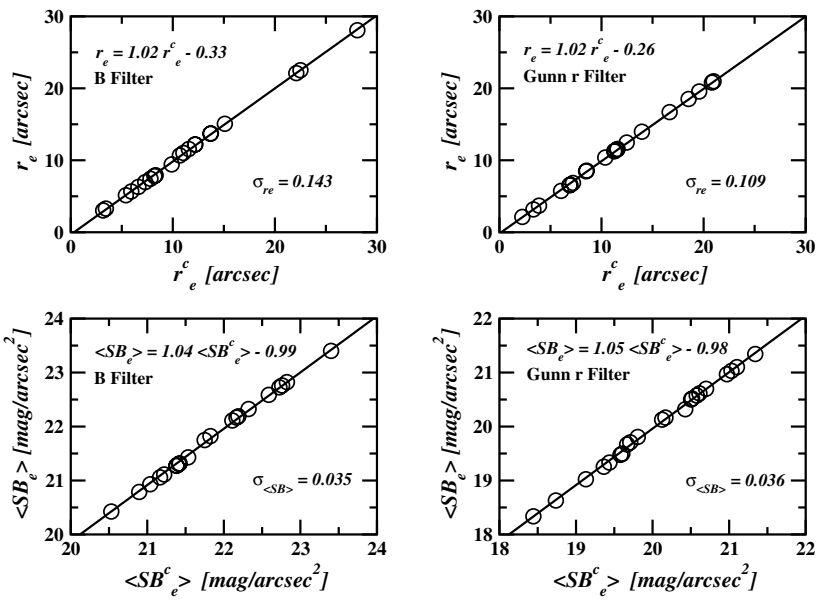

Fig. 2. Comparison of the uncorrected photometric parameters (convolved) with the corrected photometric parameters (deconvolved). The correction was carried out for seeing effects. The superscript "c" refers to the convolved value.

thus obtaining an initial value for the effective radius $r_{\mathrm{e}}$, and the mean effective surface brightness $\left\langle S B_{\mathrm{e}}\right\rangle$, with the associated errors. The effects of the seeing on the isolated $\mathrm{E}$ and $\mathrm{S} 0$ galaxies that have heliocentric velocities larger than $8000 \mathrm{~km} \mathrm{~s}^{-1}$ will be large if the size of these galaxies turns out to be smaller than 5 times the size of the seeing disk (Saglia et al. 1993). If we consider the quotient between the effective radius and the radius of the seeing disk, we find that only 10 of the 21 galaxies observed in the $B$ filter need to be corrected for seeing effects. For the Gunn $r$ filter case only 9 out of the 23 observed galaxies need to be corrected. The seeing corrections were carried out using the results by Trujillo et al. (2001a,b). Once these corrections have been applied, an important question to consider is the difference between the corrected and uncorrected photometric parameters. In Fig. 2 the comparison between the corrected and uncorrected photometric parameters is shown. For the $B$ filter the residue dispersions from the Ordinary Least Squares Fit (OLS) to the data are:

$\sigma_{S B \mathrm{e}}=0.035 \mathrm{mag} \operatorname{arcsec}^{-2}$ and $\sigma_{\text {re }}=0.143$ arcsec.

In the case of the Gunn $r$ filter dispersion values are:

$\sigma_{S B \mathrm{e}}=0.036 \mathrm{mag} \operatorname{arcsec}^{-2}$ and $\sigma_{\text {re }}=0.109$ arcsec.

As may be seen from the previous results, the seeing effect on the properties of our sample of observed galaxies is not large. This is due to the fact that the majority of galaxies are relatively near and their radii are larger than 5 times the radius of the seeing disk. For those cases for which the galaxies have small radii with respect to the size of the seeing disk, the mean correction for the mean effective surface brightness in both filters is $\sim 0.09 \mathrm{mag} \operatorname{arcsec}^{-2}$ and the correction for effective radius is $\sim 0.27$ arcsec.

The corrections for galactic extinction were calculated for each galaxy and for each filter according to the data given by Schlegel et al. (1998). Estimates for reddening $E(B-V)$ were obtained from the NED database (NASA Extragalactic Database). We also corrected for the K-effect (Pence 1976; Jørgensen et al. 1992) and cosmological dimming (Jørgensen et al. 1995). In Table A.2 (Appendix A) we show galactic extinction, K correction and cosmological dimming for each of the observed galaxies.

\subsubsection{Photometric parameters errors}

To estimate the errors of the photometric parameters, we must consider two potential sources of error: calibration errors and errors of measurement. Calibration errors were discussed in the previous section. Measurement errors belong to different categories: i) those due to isophote fitting; ii) errors due to the de Vaucouleurs profile fitting; iii) those errors associated with the "sky" brightness; and iv) the errors associated with the different corrections. The magnitude of the isophote and the de Vaucouleurs profile fitting errors come from direct application of tasks ellipse and $n$ fit $1 d$ respectively. The errors associated with sky brightness were calculated for each frame averaging the values of the mode of at least 10 empty regions in the surroundings of the galaxies and correspond to the mode standard deviation of the above-mentioned averaging process (the mean sky brightness values for both observing runs are $22.76 \pm 0.17$ and $21.33 \pm 0.17 \mathrm{mag} \operatorname{arcsec}^{-2}$ for the $B$ and the Gunn $r$ filters respectively). For the errors due to photometric corrections, we use the seeing corrections from Trujillo et al. (2001a,b) and Saglia et al. (1993), for the K correction errors we use Pence (1976) and Jørgensen et al. (1992), and those for cosmological dimming come from Jørgensen et al. (1995). In this case, we use the heliocentric Redshift $z_{\odot}$ instead of the Redshift associated with the background cosmic radiation $z_{\mathrm{CMB}}$ (where $c z_{\odot}=360 \mathrm{~km} \mathrm{~s}^{-1}$ is relative to the $\mathrm{CMB}$ in the direction $(l, b)=\left(265^{\circ}, 49^{\circ}\right)$, so that the maximun error of this approximation is of the order of $0.005 \mathrm{mag} \operatorname{arcsec}^{-2}$.

To obtain the total error we took into consideration the standard rules for error propagation. Error values for calibration and measurement were combined following the sum of the squares rule. The mean error values for $\left\langle S B_{\mathrm{e}}\right\rangle, r_{\mathrm{e}}$ and $m_{T}$ in the $B$ filter are $0.07 \mathrm{mag} \operatorname{arcsec}^{-2}, 0.6$ arcsec and 0.09 mag respectively. For the Gunn $\mathrm{r}$ filter they are $0.06 \mathrm{mag} \operatorname{arcsec}^{-2}, 0.5$ arcsec and 0.07 mag respectively. In Table A.3 we present individual estimates for the errors of each one of the photometric parameters.

\subsection{The observed catalogue of isolated $E$ and $S O$ galaxies}

In Table A.3 (Appendix A) we present the observed catalogue of the isolated $\mathrm{E}$ and $\mathrm{S} 0$ galaxies including the corrected structural parameters as well as the effective corrected colour, the average ellipticity and the total variation of the position angle for 21 galaxies in the $B$ filter and for 23 galaxies in the Gunn $r$ filter. In order to avoid the seeing effects on the average ellipticity and on the total variation of the position angle, we considered only radii larger than 5 times the seeing radius (Saglia et al. 1993). It is important to note that there are some observed galaxies whose morphological classification given by the CfA might be erroneous (for example: PGC 71203). To discuss the optical morphology as well as the fine structure (such as plumes, dust lanes or shells) of the observed galaxies, we present in Appendix B a mosaic with surface photometry information for each galaxy. Each mosaic includes; 1) image in the $B$ filter; 2) filtered image in $B$ filter; 3 ) surface brightness profile and de Vaucouleurs fit in filters $B$ and Gunn $r$; 4) colour profile $B$ - Gunn $r$; and 5) geometric profiles (radial ellipticity and position angle). Both the surface brightness profile and the colour profile are shown with no corrections.

We used our filtered images in combination with the estimated surface brightness and geometric profiles to look for morphological features in more detail and to reclassify those galaxies erroneously classified by the CfA, as well as to be able to characterise the fine structure, if present, of the observed 
galaxies (for more details see Franco-Balderas et al. 2004). The presence of fine structure might represent evidence of past merging processes or of tidal disruption due to interactions with small galaxies and/or acquisition events (Binney \& Petrou 1985; Nieto \& Bender 1989; Schweizer et al. 1990; Reduzzi et al. 1996; Reda et al. 2004). A detailed study of the fine structure present in the galaxies observed, allowed us to characterise better our sample of isolated galaxies. The results are presented in Table A.4 (Appendix A).

According to the new morphological classification presented in Table A.4, galaxies PGC 13088, PGC 35545, PGC 68438 and PGC 71203 are late-type galaxies and, therefore, were not be included in the KR analysis. For galaxies PGC 5660, PGC 10538 and PGC 32231 we found possible evidence of structures. However, it is difficult to determine whether these are spurious structures, spiral arms or rests of recent merger/acquisition events. To be on the safe side, we excluded these galaxies from the KR analysis. It is important to mention that the deep and filtered images, the surface brightness profiles as well as the geometric profiles of the bona fide ETG do not show signs of obvious fine structures, except in the case of PGC 31768, so this galaxy was not included in the KR analysis either. On the other hand, another one of the observed galaxies (PGC 69782) has a very bright star in one of its edges. The presence of this star produces very large errors in the Gunn $r$ filter photometry. This galaxy was not included in the calculations of the KR (Gunn $r$ filter) either, so the final sample used for these calculations contained 15 galaxies in the $B$ filter and 14 in the Gunn $r$ filter.

The sample of bona fide isolated ETG observed in the $B$ filter comprises 3 elliptical galaxies, and $13 \mathrm{~S} 0$ galaxies distributed in an absolute magnitude range $-18.7 \geq M_{B}>-21.0$, the ratio of elliptical galaxies with respect to the total observed $\mathrm{E}+\mathrm{S} 0$ is approximately 19\%. In the case of the Gunn $r$ filter we have one elliptical galaxy and 14 S0 galaxies distributed in the absolute magnitude range $-20.8 \geq M_{\mathrm{Gr}}>-23.0$ so that the ratio of elliptical galaxies with respect to the total number observed is approximately 7\%. Only one galaxy (PGC 31768) shows evidence of fine structure (a weak luminous shell, see Table A.4 for details) and represents $7 \%$ of the total bona fide ETG sample observed. This percentage is lower than that found for other samples in the literature, for example Reduzzi et al. (1996) and Reda et al. (2004) find that approximate $40 \%$ of their isolated galaxies show evidence of a past interaction/merger event.

\subsection{Comparison between the observed photometry and data from the literature}

Since the data reported in the LEDA database are homogeneous, i.e. expressed in the same photometric system, we used these data to establish a comparison with our observed data. It is to be expected that there will be differences between our data and those from LEDA since the methods to measure the total magnitudes and the photometric systems are different. Besides, the errors associated with the LEDA magnitudes are relatively large $(\langle\varepsilon\rangle \sim 0.4 \mathrm{mag})$.

In Fig. 3 we present the comparison for the uncorrected magnitudes in the $B$ filter. The dispersion turns out to be of the order $0.325 \mathrm{mag}$. For the Gunn $r$ filter, there is, in the LEDA, information only for two of our galaxies. With these numbers we find that the mean difference value is $0.18 \mathrm{mag}$. As mentioned in Sect. 3.1, in the literature there are 7 galaxies with information of structural parameters $\left(\left\langle S B_{\mathrm{e}}\right\rangle\right.$ and $\left.m_{T}\right)$. Nevertheless, only two of them (NGC 3332, NGC 3716) were observed by us during the observing seasons described above. Given that the information

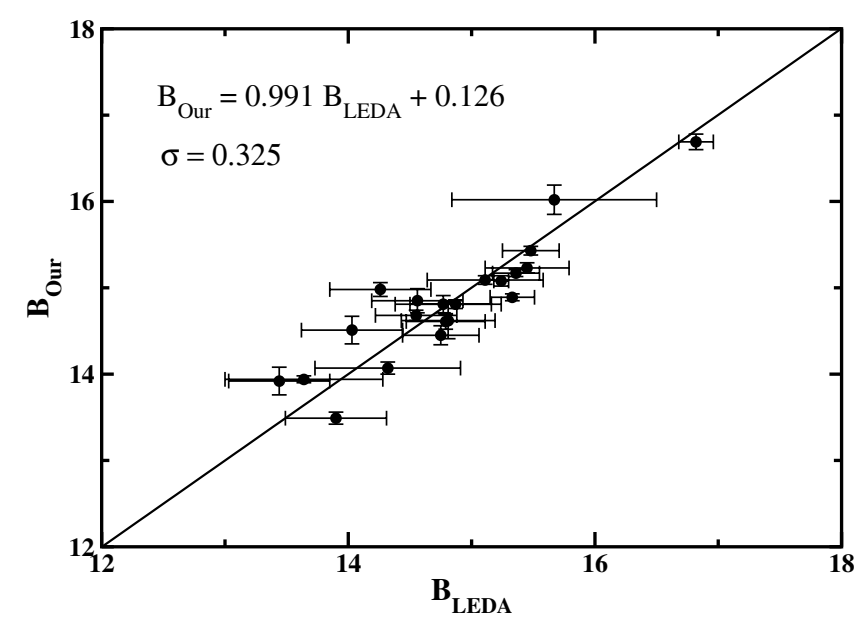

Fig. 3. Comparison between the apparent uncorrected $B$ magnitudes obtained in this work, and those given in the LEDA database. The residue dispersion is $0.325 \mathrm{mag}$.

in the literature (Alonso et al. 2003) corresponds to the $R_{\mathrm{c}}$ filter (Kron-Cousins), it was necessary to transform to the Gunn $r$ filter using the following equation: Gunn $r-R_{\mathrm{c}}=0.354$ (Jørgensen \& Franx 1994; Milvang-Jensen 1997). Using this information, and considering that these authors do not use the same methods for photometric and cosmological corrections (see Sects. 4.1 and 4.2) we find that the mean difference between the effective surface brightness in the literature and ours is $0.19 \mathrm{mag} \operatorname{arcsec}^{-2}$.

Our sample of observed E and S0 galaxies has an effective mean colour $(B-\text { Gunn } r)_{\mathrm{e}}=1.40 \pm 0.2$. Prugniel \& Heraudeau $(1996)$ report $(B-R)_{\mathrm{e}}=1.52 \pm 0.2$ for $\mathrm{S} 0$ and $(B-R)_{\mathrm{e}}=1.57 \pm 0.2$ for ellipticals. On the other hand Reda et al. (2004) report $(B-$ $R)_{\mathrm{e}}=1.54 \pm 0.14$ for a sample of isolated ETG. If we consider the differences due to the filters and the fact that the literature data do not use the same photometric and cosmological corrections (galactic extinction, $\mathrm{K}$ and cosmological dimming corrections) as those used in this paper; then our colours are consistent with those in the literature within the associated errors.

\section{Comparison-samples of ETG from the literature}

\subsection{The sample of ETG in Coma cluster}

In what follows, the Coma cluster is used as the low-redshift $(z=0.024)$ and high-density reference for our study of changes in the KR as a function of gravitational interactions. We used a sample of $116 \mathrm{E}$ and S0 galaxies of the Coma Cluster, which has photometry in the Gunn $r$ filter from Jørgensen et al. (1995), Milvang-Jensen (1997) and Jørgensen et al. (1999). According to Jørgensen et al. (1999) the sample is $93 \%$ complete to a magnitude limit of $m_{\mathrm{Gr}}=15.05^{\mathrm{m}}$ in Gunn $r$, equivalent to $M_{\mathrm{Gr}}=-20.75$ (for a distance modulus of $35.8 \mathrm{mag}$ ). The range of absolute magnitudes covered by this sample is $-20.4 \geq M_{\mathrm{Gr}}>$ -24.9. The photometric and the cosmological corrections for this sample are performed by the authors following the same methodology utilised for the sample of isolated ETG, except for the case of galactic extinction, where the authors used the determinations given by Burstein \& Heiles (1984). If we consider the sample of observed isolated ETG and carry out the galactic extinction corrections from Schlegel (1998) as well as those from Burstein \& Heiles (1984), the mean difference found is of the order 0.134 mag for the $B$ filter and of the order 0.077 mag 
for the Gunn $r$ filter, so these differences need to be considered when comparisons are carried out.

It is important to mention that the $f$ parameter in Coma, according to Varela et al. (2004) is in the range $-4.5<f<0.25$.

\subsection{The sample of perturbed SO galaxies}

Varela et al. (2004) have defined and compiled a sample of 130 perturbed S and S0 galaxies. This sample was extracted from the CfA catalogue within a volume bound by $c z \leq 5000 \mathrm{~km} \mathrm{~s}^{-1}$ $(z \leq 0.017)$, galactic latitude $|b| \geq 40^{\circ}$, declination $-2.5 \leq \delta \leq$ 90 , and was defined comprising galaxies with confirmed companions satisfying the isolation parameter, $f>-2$. According to Varela et al. (2004), the criterion used on the $f$ parameter to establish whether a galaxy is being perturbed or not is based on theoretical results from Athanassoula (1984), Byrd \& Howard (1992) and Icke (1985), from which they get that $f>-2$ so that there are measurable effects on the properties of a given galaxy.

The Varela et al. (2004) sample gives neither information for the effective parameters nor information on the environment within which the sample galaxies are embedded. We therefore undertook an exhaustive literature search to obtain information for these effective parameters and the environment for the S0 galaxies in this sample. Our findings are as follows:

- 12 S0 galaxies have effective radius and mean effective surface brightness information for the $B$ filter (Prugniel \& Simien 1996; Alonso et al. 2003). All 12 galaxies are located within dense environments such as groups or clusters.

- 18 S0 galaxies have information for both the effective radius and the mean effective surface brightness for the $R_{\mathrm{c}}$ filter (Kron-Cousins system) (Prugniel \& Simien 1996; Alonso et al. 2003). Only $90 \%$ of these 18 galaxies have information about their environment, and of these, all belong to groups or clusters.

An important characteristic of S0 galaxies is that, in general, the structural properties of their bulges show approximately the same homogeneity as those of elliptical galaxies. Due to this fact, and because KR uses effective parameters, we use the sample of perturbed S0 galaxies as a reference case of strong gravitational interaction in our study of changes in the KR as a function of gravitational interaction.

In order to be able to carry out proper comparisons between the samples of isolated and cluster galaxies with that of perturbed galaxies, it is necessary that all data are expressed in the same filter. The $R_{\mathrm{c}}$ was transformed to the Gunn $r$ filter using the following relationship: Gunn $r-R_{\mathrm{c}}=0.354 \mathrm{mag}$ (Jørgensen \& Franx 1994; Milvang-Jensen 1997). The data must also be corrected for seeing effects, galactic extinction, $\mathrm{K}$ correction and cosmological dimming. The data from Prugniel \& Simien (1996) and Alonso et al. (2003) consider all the corrections mentioned above, even though the correction for extinction and the K correction were done using Burstein \& Heiles (1984) data and those from Davis et al. (1985) respectively. The cosmological corrections were done using $(1+z)^{4}$. If we consider the sample of observed isolated ETG, the mean difference between the corrections applied by us and those applied by Prugniel \& Simien (1996) and Alonso et al. (2003) is of the order $0.158 \mathrm{mag}$ in the $B$ filter and of the order 0.101 in the Gunn $r$ filter.

Tables A.5 and A.6 (Appendix A) present photometric data from the literature for the sample of perturbed SO galaxies, as well as the type of environment within which they are embedded.

\section{The Kormendy relation}

A relevant projection of the FP is the correlation between $r_{\mathrm{e}}$ and $\left\langle S B_{\mathrm{e}}\right\rangle$, which is also known as the KR:

$\left\langle S B_{\mathrm{e}}\right\rangle=\alpha+\beta \log \left(r_{\mathrm{e}}\right)$,

This relation is particularly useful in the study of scale relations due to the fact that it only requires photometric information.

A large number of studies has demonstrated that cluster ETG define KR with an intrinsic dispersion of approximately 0.4 mag $\operatorname{arcsec}^{-2}$ in $\left\langle S B_{\mathrm{e}}\right\rangle$ (Hamabe \& Kormendy 1987; Hoessel et al. 1987; Sandage \& Lubin 1991; Sandage \& Perelmuter 2001). However, for the case of isolated ETG there are practically no studies regarding this relation. Some authors report that other relations, such as, the FP, or the metallicity-velocity dispersion relation for isolated ETG are not significantly different from those obtained in clusters (Ziegler et al. 1999; Evstigneeva et al. 2002; Reda et al. 2005). These studies have been undertaken through numerical simulations and/or the isolation criteria which have been used to define their sample have not been very strict, so the occurrence of physical processes such as tidal interactions or ram pressure, cannot be excluded. Our isolation criteria, as we discussed above, are very strict. We are, therefore, positive that the effects of none of the mentioned physical processes will have any bearing on our results.

From the surface photometry information for the isolated, Coma and perturbed galaxies we calculate the coefficients $\alpha$ and $\beta$ of the KR using the $B C E S_{\text {Bis }}$ fitting method (Isobe et al. 1990; Akritas \& Bershady 1996), as well as the intrinsic dispersion $\left(\sigma_{\text {Int }}\right)$ in $\left\langle S B_{\mathrm{e}}\right\rangle$ (following the method of La Barbera et al. 2003) and the correlation coefficient $R$ of the fit. The errors in the coefficients were calculated following Akritas \& Bershady (1996) in $1 \sigma$ intervals. In Table 2 we present our results for the B and the Gunn $r$ filters. It is important to mention that the surface photometry of perturbed galaxies in the $B$ filter does not include the errors in the $\left\langle S B_{\mathrm{e}}\right\rangle$ and $r_{\mathrm{e}}$, so that the intrinsic dispersion in $\left\langle S B_{\mathrm{e}}\right\rangle$ was calculated using typical values for the error residue dispersion in the Gunn $r$ filter; $\delta_{\langle S B \mathrm{e}\rangle}=0.09$ and $\delta_{\langle\mathrm{re}\rangle}=0.03$.

In Fig. 4 we show the distribution of the galaxies in the $\log \left(r_{\mathrm{e}}\right)-\left\langle S B_{\mathrm{e}}\right\rangle$ plane. The continuous line represents the $B C E S_{\text {Bis }}$ fit.

\subsection{Comparison of Kormendy relation of ETG under different gravitational interaction}

As we have seen previously, the different samples of ETG compiled in this paper cover a relatively ample gravitational interaction range, from isolated galaxies $(f \leq-4.5)$, passing through cluster galaxies $(-4.5<f<0.25)$ to galaxies with confirmed companions $(f>-2)$. So that the comparisons that we will establish, in the following paragraphs, between their properties will be relevant.

When we compare the coefficients $\beta$ of the KR for isolated, Coma and perturbed galaxies, we find that there are no significant differences among them, in fact, the differences are similar in size to the associated errors. The difference in the zero-point might be explained by the fact that the samples do not cover the same Redshift interval (La Barbera et al. 2003) and that some of the corrections (extinction correction, $\mathrm{K}$ correction, cosmological dimming) used by the authors who obtained the sample were not exactly the same as those used in this study for the sample of isolated galaxies. In the case of intrinsic dispersion, the differences we found seem to depend on the magnitude range within 
Table 2. KR parameters ( $B$ and Gunn $r$ filters) for the isolated, Coma and perturbed galaxies. $N$ is the number of galaxies in the sample and MI is the absolute magnitude interval within as the galaxies are distributed.

\begin{tabular}{|c|c|c|c|}
\hline & \multicolumn{3}{|c|}{$B$ Filter } \\
\hline KR coefficients & Isolated & Coma & Perturbed \\
\hline$\beta$ & $3.056 \pm 0.267$ & - & $3.406 \pm 0.096$ \\
\hline$\alpha$ & $19.321 \pm 0.157$ & - & $19.471 \pm 0.095$ \\
\hline$\sigma_{\text {Int }}$ & 0.379 & - & 0.303 \\
\hline$R$ & 0.835 & - & 0.949 \\
\hline$N$ & 15 & - & 12 \\
\hline MI & $-18.7 \geq M_{B}>-21.1$ & - & $-19.0 \geq M_{B}>-20.9$ \\
\hline \multicolumn{4}{|c|}{ Gunn $r$ filter } \\
\hline$\beta$ & $3.387 \pm 0.189$ & $3.232 \pm 0.159$ & $3.317 \pm 0.146$ \\
\hline$\alpha$ & $17.636 \pm 0.136$ & $18.056 \pm 0.087$ & $17.895 \pm 0.103$ \\
\hline$\sigma_{\text {Int }}$ & 0.381 & 0.536 & 0.389 \\
\hline$R$ & 0.892 & 0.786 & 0.925 \\
\hline$N$ & 14 & 116 & 18 \\
\hline MI & $-20.8 \geq M_{\mathrm{Gr}}>-23.0$ & $-20.4 \geq M_{\mathrm{Gr}}>-24.9$ & $-20.3 \geq M_{\mathrm{Gr}}>-22.6$ \\
\hline
\end{tabular}
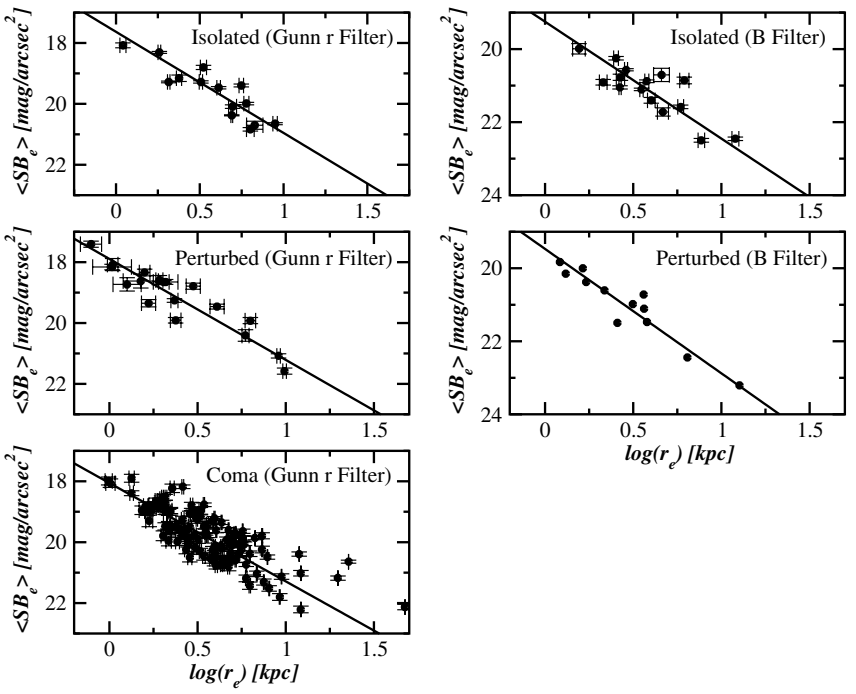

Fig. 4. KR of isolated, perturbed and Coma galaxies. The continuous line represents the $B C E S_{\text {Bis }}$ fit. The surface photometry of perturbed galaxies in the $B$ filter does not include the errors in the $\left\langle S B_{\mathrm{e}}\right\rangle$ and $r_{\mathrm{e}}$, so that the intrinsic dispersion in $\left\langle S B_{\mathrm{e}}\right\rangle$ was calculated using typical values for the error residue dispersion in the Gunn $r$ filter; $\delta_{\langle S B e\rangle}=0.09$ and $\delta_{\langle r e\rangle}=0.03$.

which the galaxies are found and not on the degree of gravitational interaction to which they are subjected. As may be seen in Table 2, the intrinsic dispersion of the samples is approximately the same within similar magnitude ranges, and is larger when the magnitude range is larger. The dependence of the intrinsic dispersion on the magnitude range may be checked with the Coma Cluster data by reducing the magnitude interval to a size similar to that of the other samples. In Table 3 we see that, for the Coma Cluster, the intrinsic dispersion diminishes when we diminish the magnitude range and that this dispersion value is similar to that for the other samples provided the magnitude range is similar (see Tables 2 and 3). This dependence might be due to the fact that galaxy distribution on the $\left\langle S B_{\mathrm{e}}\right\rangle-r_{\mathrm{e}}$ plane depends on luminosity, i.e. brighter galaxies shift to the large size, lower surface brightness region. This shift produces a depopulation of the region that corresponds to equal luminosity galaxies but with smaller sizes and higher surface brightness values (Varela 2004; D'Onofrio et al. 2006). In other words, equal luminosity galaxies are distributed approximately in parallel bands in the $\left\langle S B_{\mathrm{e}}\right\rangle-r_{\mathrm{e}}$
Table 3. Intrinsic dispersion and correlation coefficient of the KR for the Coma galaxies (Gunn $r$ filter) in different magnitude intervals. MI is the absolute magnitude interval within as the galaxies are distributed and $\mathrm{N}$ is the number of galaxies in the sample. The interval $-20.4 \geq$ $M_{\mathrm{Gr}}>-24.9$ is that of the original sample and the interval $-20.4 \geq$ $M_{\mathrm{Gr}}>-22.7$ is similar to that of the comparison samples (perturbed and isolated galaxies).

\begin{tabular}{cccc}
\hline \hline \multicolumn{3}{c}{ Coma (Gunn $r$ Filter) } \\
\hline MI & $-20.4 \geq M_{\mathrm{Gr}}>-24.9$ & $-20.4 \geq M_{\mathrm{Gr}}>-22.7$ & $-20.4 \geq M_{\mathrm{Gr}}>-21.4$ \\
$\sigma_{\mathrm{Int}}$ & 0.536 & 0.458 & 0.259 \\
$R$ & 0.786 & 0.925 & 0.950 \\
$N$ & 116 & 103 & 50 \\
\hline MI & $-21.0 \geq M_{\mathrm{Gr}}>-24.9$ & $-22.0 \geq M_{\mathrm{Gr}}>-24.9$ & $-23.0 \geq M_{\mathrm{Gr}}>-24.9$ \\
$\sigma_{\mathrm{Int}}$ & 0.513 & 0.329 & 0.335 \\
$R$ & 0.817 & 0.908 & 0.887 \\
$N$ & 89 & 38 & 6 \\
\hline \multicolumn{4}{r}{}
\end{tabular}

plane, so the larger the magnitude interval of a galaxy sample is, the larger the range in the $\left\langle S B_{\mathrm{e}}\right\rangle-r_{\mathrm{e}}$ plane will be, and so the dispersion of the observed data will be larger.

From the previously mentioned results, we may infer that the fundamental parameters of the galaxies used in calculating the KR are not sensitive to effects related to gravitational interactions. One possible explanation is that the gravitational interactions are "erased" or "absorbed" rapidly by the galaxies, leaving little or no sign of the interaction event. Another explanation might be that the majority of the interactions will perturb only the external parts of the galaxies (or only external parts will keep memory of the perturbations), which are not represented in the KR.

This result agrees with some recent papers which deal with structural properties of ETG (de la Rosa et al. 2001; Treu et al. 2001; Evstigneeva et al. 2002; Gonzáles-García \& van Albada 2003; Denicoló et al. 2005; Reda et al. 2005). For example Evstigneeva et al. (2002) have found, using numerical simulations, that structural parameters of ETG are stable to gravitational perturbations. These parameters experiment changes during close encounters, but within a very short time interval $\left(\sim 10^{7}-10^{8}\right.$ years), and they change just before the final merging process. Moreover, the amplitude of the changes is comparable to the dispersion in the values of the observed structural relations.

Therefore, if the structural parameters involved in the KR are not sensitive to effects due to gravitational interactions, this implies that ETG present in any type of environment may be characterised by these photometric parameters. 
Finally if we compare the coefficients of the KR for the samples in the $B$ and Gunn $r$ filters (see Table 2) we see that there are no significant differences, i.e. the KR does not depend on wavelength. This result must be treated with caution since we do not count, as yet, with a sufficiently extended wavelength range.

\section{Summary and conclusions}

1. We defined a sample of 82 isolated ETG using very strict isolation criteria and trying to avoid objects with interactions or merger/acquisition events in the recent past.

2. Using photometric observations, we obtained the structural parameters for 21 galaxies in the $B$ filter and for 23 galaxies in the Gunn $r$ filter of the sample of isolated ETG.

3. We also studied a sample of 116 Coma cluster ETG in the Gunn $r$ filter (Jørgensen et al. 1999; Milvang-Jensen 1997) and a sample of perturbed S0 galaxies from Varela et al. (2004), for which no information is given for effective parameters or type of environment. A literature search for the perturbed $\mathrm{S} 0$ galaxies resulted in structural parameter values for 12 galaxies in the $B$ filter and for 18 in the $R_{\mathrm{c}}$ filter. Only $93 \%$ of these galaxies have information about their environment, and of these, all belong to groups or clusters.

4. We obtained the KR for the isolated as well as Coma and perturbed ETG samples, and compared them. Our comparisons show that:

- No significant differences in the beta $(\beta)$ coefficient are found for the different galaxy samples.

- There is a difference in the zero-point which may be explained by noting that all the samples do not cover the same Redshift interval (La Barbera et al. 2003) and that the corrections applied (extinction correction,
K correction, cosmological dimming) are not exactly the same for all the samples.

- The intrinsic dispersion of the KR depends on the magnitude range of the galaxy sample and not on the gravitational interaction to which the galaxies in each sample are subjected. This fact might be explained by saying that galaxy distribution on the $\left\langle S B_{\mathrm{e}}\right\rangle-r_{\mathrm{e}}$ plane depends on luminosity (see Sect. 5.1 for full details).

5. The KR appears to be wavelength independent. Nonetheless, in order to confirm this result, observations in a larger wavelength range are required.

6. ETG contained within any type of environment may be characterised by the same set of structural parameters since the structural parameters involved in the KR are not sensitive to effects due to gravitational interactions.

It is, however, necessary to consider larger samples to establish these results as conclusive. The main purpose of our future research is to obtain the structural parameters for the entire sample of isolated ETG and supplement this information with spectroscopic parameters to extend the study to the rest of the structural relations (Faber-Jackson relation, $\mathrm{Mg}_{2}$-velocity dispersion relation and the FP).

Acknowledgements. We would like to thank Consejo Nacional de Ciencia y Tecnología (México) for the Ph.D. fellowship number 132526, the Spanish Ministerio de Educación y Ciencia for the grant PNAYA2006-14056 and Instituto de Matemáticas y Física Fundamental (Madrid España) for logistic support. We would also like to record our obligation to Jesús Varela, Margarita Rosado, Lorena Arias and Patricia Ambrosio for help with the observations. Last but not least, we would like to express our deepest appreciation to an anonymous referee whose comments and suggestions greatly improved the presentation of this paper.

\section{Appendix A: Catalogues of early-type galaxies}

Table A.2. Journal of observations and photometric corrections. Column (1) PGC Name. Column (2) exposure time in seconds, $B$ filter. Column (3) seeing in $B$ filter. Column (4) exposure time in seconds, Gunn $r$ filter. Column (5) seeing in Gunn $r$ filter. Column (6) galactic extintion in $B$ filter (Schlegel et al. 1998). Column (7) galactic extintion in Gunn $r$ filter (Schlegel et al. 1998). Column (8) K correction in $B$ filter (Pence 1976). Column (9) K correction in Gunn $r$ filter (Jørgensen et al. 1992). Column (10) cosmological dimming (Jørgensen et al. 1995).

\begin{tabular}{|c|c|c|c|c|c|c|c|c|c|}
\hline PGC Name & $\begin{array}{c}T_{B} \\
\mathrm{~S} \\
(2)\end{array}$ & $\begin{array}{c}F W H M_{B} \\
\prime \prime \\
\text { (3) }\end{array}$ & $\begin{array}{c}T_{\text {Gunn } r} \\
\text { S } \\
(4)\end{array}$ & $\begin{array}{c}F W H M_{\mathrm{Gunn} r} \\
\text { " } \\
\text { (5) }\end{array}$ & $\begin{array}{c}A_{B} \\
\mathrm{mag} \\
(6)\end{array}$ & $\begin{array}{c}A_{\mathrm{Gunn} r} \\
\text { mag } \\
(7)\end{array}$ & $\begin{array}{c}K_{B} \\
\text { mag } \\
(8)\end{array}$ & $\begin{array}{c}K_{\mathrm{Gunn} r} \\
\text { mag } \\
(9)\end{array}$ & $\begin{array}{c}D \\
\text { mag } \\
(10)\end{array}$ \\
\hline 5381 & $2 \times 1800$ & 1.0 & $2 \times 600$ & 1.0 & 0.077 & 0.047 & 0.152 & 0.035 & 0.138 \\
\hline 5655 & $2 \times 1000$ & 1.5 & $2 \times 300$ & 1.5 & 0.129 & 0.078 & 0.081 & 0.019 & 0.076 \\
\hline 5660 & $2 \times 1200$ & 1.3 & $2 \times 500$ & 1.3 & 0.068 & 0.041 & 0.084 & 0.019 & 0.079 \\
\hline 7658 & $2 \times 1200$ & 1.5 & $2 \times 480$ & 1.0 & 0.266 & 0.161 & 0.091 & 0.021 & 0.085 \\
\hline 7820 & $2 \times 1600$ & 1.3 & $2 \times 300$ & 1.0 & 0.183 & 0.111 & 0.084 & 0.019 & 0.079 \\
\hline 10538 & $2 \times 1000$ & 1.3 & $2 \times 360$ & 1.2 & 0.749 & 0.451 & 0.118 & 0.027 & 0.109 \\
\hline 11254 & $2 \times 1200$ & 1.0 & $2 \times 360$ & 1.0 & 0.991 & 0.596 & 0.097 & 0.023 & 0.091 \\
\hline 13088 & & & $2 \times 360$ & 1.2 & & 0.544 & & 0.024 & 0.094 \\
\hline 28009 & $2 \times 1200$ & 1.2 & $2 \times 360$ & 1.4 & 0.177 & 0.106 & 0.076 & 0.018 & 0.072 \\
\hline 31768 & $2 \times 720$ & 1.2 & $2 \times 240$ & 1.2 & 0.139 & 0.083 & 0.089 & 0.021 & 0.084 \\
\hline 32231 & & & $2 \times 300$ & 1.0 & & 0.069 & & 0.021 & 0.084 \\
\hline 33460 & $2 \times 1000$ & 1.0 & $2 \times 300$ & 1.0 & 0.083 & 0.049 & 0.097 & 0.023 & 0.090 \\
\hline 33917 & $2 \times 1200$ & 1.1 & $2 \times 360$ & 1.1 & 0.205 & 0.124 & 0.118 & 0.027 & 0.109 \\
\hline 35545 & $2 \times 1200$ & 1.0 & $2 \times 360$ & 1.0 & 0.092 & 0.054 & 0.102 & 0.024 & 0.095 \\
\hline 36946 & $2 \times 1200$ & 1.5 & & & 0.089 & & 0.086 & & 0.081 \\
\hline 37105 & & & $2 \times 480$ & 1.5 & & 0.085 & & 0.023 & 0.092 \\
\hline 170386 & $2 \times 1000$ & 1.4 & $2 \times 400$ & 1.3 & 0.164 & 0.096 & 0.153 & 0.035 & 0.139 \\
\hline 68438 & $2 \times 1500$ & 1.3 & $2 \times 400$ & 1.4 & 0.208 & 0.124 & 0.139 & 0.032 & 0.127 \\
\hline 68934 & $2 \times 1000$ & 1.3 & $2 \times 400$ & 1.3 & 0.142 & 0.085 & 0.117 & 0.027 & 0.108 \\
\hline 133427 & $2 \times 1300$ & 1.6 & $2 \times 400$ & 1.6 & 0.078 & 0.047 & 0.129 & 0.029 & 0.118 \\
\hline 69548 & $2 \times 1000$ & 1.2 & $2 \times 200$ & 1.2 & 0.321 & 0.192 & 0.112 & 0.026 & 0.104 \\
\hline 69782 & $2 \times 400$ & 1.6 & $2 \times 100$ & 1.4 & 0.151 & 0.091 & 0.133 & 0.031 & 0.122 \\
\hline 70499 & $2 \times 1000$ & 1.3 & $2 \times 230$ & 1.3 & 0.180 & 0.109 & 0.110 & 0.026 & 0.102 \\
\hline 71203 & $2 \times 1500$ & 1.0 & $2 \times 550$ & 1.0 & 0.126 & 0.075 & 0.091 & 0.021 & 0.085 \\
\hline
\end{tabular}




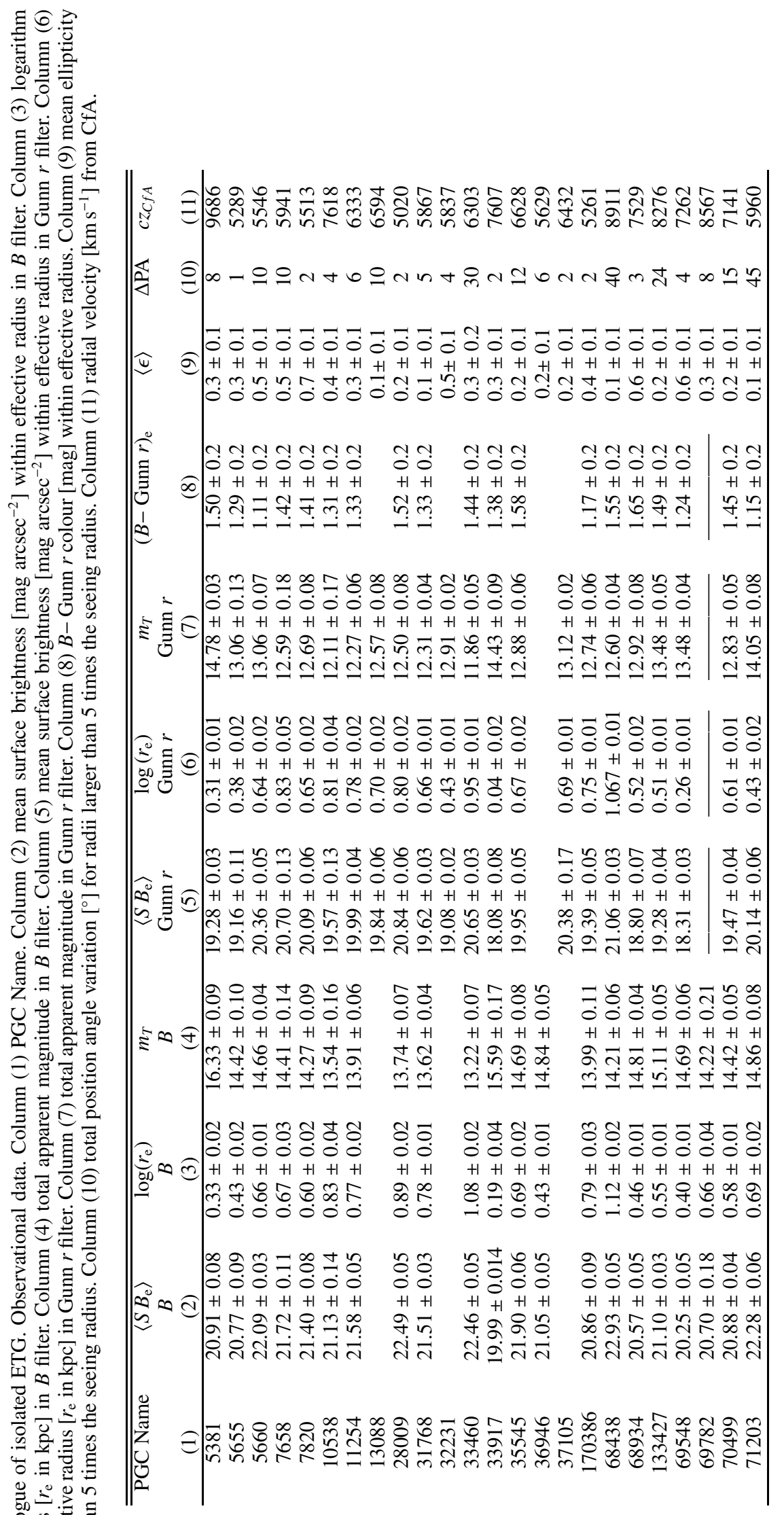


Table A.4. Catalogue of isolated ETG. Morphological features. Column (1) PGC Name. Column (2) Hubble-type from CfA. Column (3) Hubbletype from this work. Column (4) notes about surface photometry and isolation. AC $=$ Apparent companion (possible companion that placed at the distance of the galaxy would have very small size $(r<2 \mathrm{kpc})$, very low luminosity $(M>-12)$ or projected galaxy with redshift difference $\left.\Delta v>500 \mathrm{~km} \mathrm{~s}^{-1}\right) . \mathrm{HG}=$ Classified as an isolated galaxy by Haynes \& Giovanelli (1984). CMZ = Classified as an isolated galaxy by Colbert et al. (2001).

\begin{tabular}{|c|c|c|c|}
\hline $\begin{array}{l}\text { PGC Name } \\
\text { (1) }\end{array}$ & $\begin{array}{c}\text { Hubble-type (CfA) } \\
\text { (2) }\end{array}$ & $\begin{array}{c}\text { Hubble-type (this work) } \\
\text { (3) }\end{array}$ & $\begin{array}{l}\text { Notes } \\
\text { (4) }\end{array}$ \\
\hline 5381 & $\mathrm{~S} 0$ & S0 & $\mathrm{AC}$ \\
\hline 5655 & S0 & S0 & $\mathrm{AC}$ \\
\hline 5660 & So & Sa? & Twisted isophotes. Spurious structure caused by the twist? \\
\hline 7658 & S0 & S0/a & Twisted isophotes. AC \\
\hline 7820 & $\mathrm{~S} 0 / \mathrm{a}$ & S0/a & \\
\hline 10538 & S0/a & $\mathrm{Sa} ?$ & Spiral arms or reminiscent of past merging? \\
\hline 11254 & S0 & So & $\mathrm{AC}$ \\
\hline 13088 & S0 & Sab & HG \\
\hline 28009 & S0 & So & $\mathrm{AC}$ \\
\hline 31768 & So & So & Extra tidal light. Weak light shell to the northeast. CMZ \\
\hline 32231 & $\mathrm{~S} 0 / \mathrm{a}$ & Sa? & Spiral arms or reminiscent of past merging? \\
\hline 33460 & S0 & So & \\
\hline 33917 & S0 & S0 & \\
\hline 35545 & So & Sab & \\
\hline 36946 & $\mathrm{E}$ & $\mathrm{E} / \mathrm{S} 0$ & \\
\hline 37105 & S0 & S0 & $\mathrm{AC}$ \\
\hline 170386 & So & S0 & $\mathrm{AC}$ \\
\hline 68438 & S0/a & Sab & Large variation of the position angle. AC \\
\hline 68934 & S0 & So & $\mathrm{AC}$ \\
\hline 133427 & So & $\mathrm{E}$ & Twisted isophotes \\
\hline 69548 & S0 & S0 & \\
\hline 69782 & E/S0 & $\mathrm{E} / \mathrm{S} 0$ & Nearby bright star \\
\hline 70499 & S0 & SB0 & Bar. AC \\
\hline 71203 & S0 & Sbc & Large variation of the position angle. Nearby bright star \\
\hline
\end{tabular}




\section{Appendix B: B and Gunn $r$ surface photometry of isolated early-type galaxies}
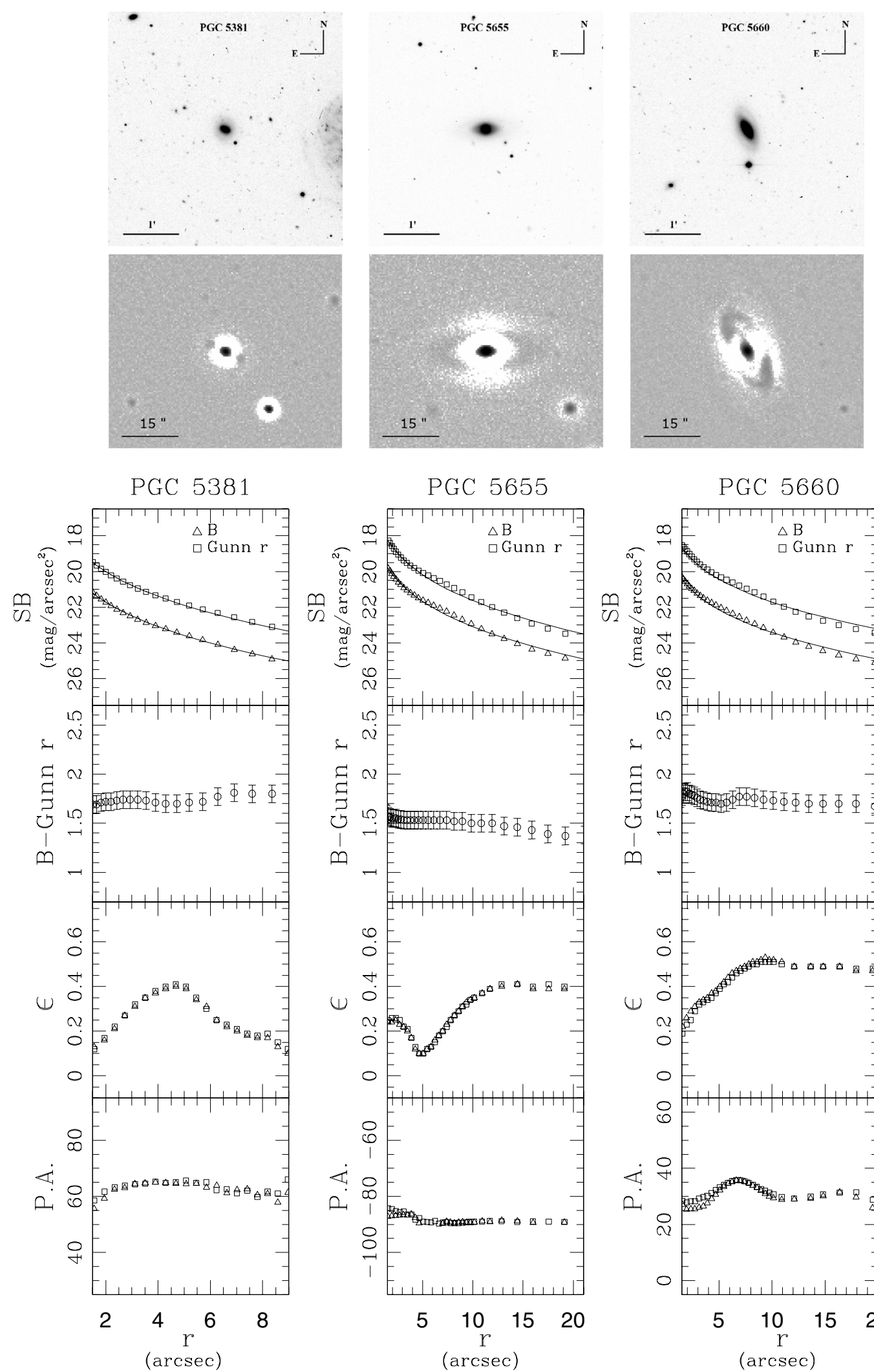

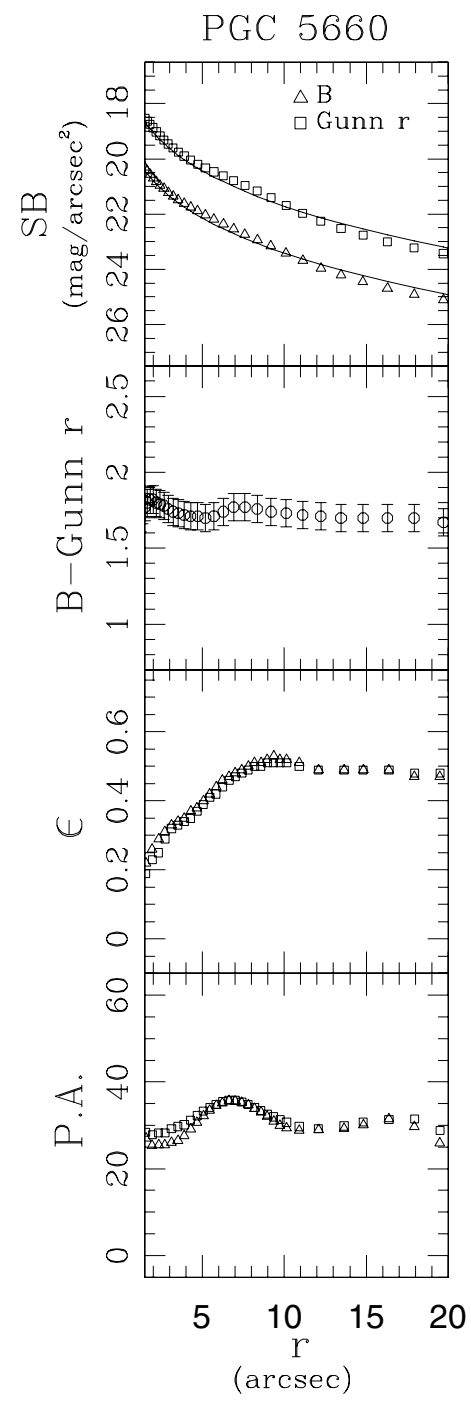

Fig. B.1. Each panel contains: image in $B$ filter, filtered image in $B$ filter, $B$ and Gunn $r$ filter surface brightness (the continuous line represents the de Vaucouleurs fit), $B$ - Gunn $r$ colour, ellipticity $(\epsilon)$ profile and position angle (PA) profile. Both the surface brightness profile and the colour profile are shown with no corrections. The $B$ and Gunn $r$ filter data are represented by squares and triangles respectively. 


\section{References}

Aars, C. E., Marcum P. M., \& Fanelli, M. N. 2001, AJ, 122, 2923

Athanassoula, E. 1984, Phys. Rep., 114, 321

Akritas, M., \& Bershady, M. 1996, ApJ, 470, 706

Alonso, M. V., Bernardi, M., da Costa, L. N., et al. 2003, AJ, 125, 2307

Bahcall, N. A. 1977, ARA\&A, 15, 505

Balogh, M. L., Morris, S. L., Yee, H. K. C., Carlberg, R. G., \& Ellingson E. 1997, ApJ, 488, L75

Baugh, C. M., Cole, S., Frenk, C. S., \& Lacey C. G. 1998, ApJ, 498, 504

Bernardi, M., Sheth, R. K., Annis, J., et al. 2003, AJ, 125, 1849

Berlind, A. A., Blanton, M. R., Hogg, D. W., et al. 2005, ApJ, 629, 625

Binney, J., \& Petrou, M. 1985, MNRAS, 214, 449

Blanton, M. R., Hogg, D. W., Bahcall, N. A., et al. 2003, ApJ, 594, 186

Burstein, D., \& Heiles, C. 1984, ApJS, 89, 131

Byrd, G. G., \& Howard, S. 1992, AJ, 103, 1089

Couch, W. J., Balogh, M. L., Bower, R. G., et al. 2001, ApJ, 549, 820

de la Rosa, I. G., de Carvalho R. R., \& Zepf, S. E. 2001, AJ, 122, 93

de Carvalho, R. R., \& Djorgovski, S. 1992, ApJ, 389, L49

Colbert, J. W., Mulchaey, J. S., \& Zabludoff, A. I. 2001, AJ, 121, 808

Dekel, A., \& Ostriker, J. P. 1999, Formation of the structure in the Universe (Cambridge University Press) 142

Denicoló, G., Terlevich, R., Terlevich, E., et al. 2005, MNRAS, 356, 1440

Davis, L., Cawson, M., Davis, R. L., \& Illingworth, G. D. 1985, AJ, 90, 169

Djorgovski, S., \& Davis, M. 1987, ApJ, 313, 59

D’Onofrio, M., Valentinuzzi, T., Secco, L., Caimmi, R., \& Bindoni, D. 2006, NewAR, 50, 447

Dressler, A. 1980, ApJ, 236, 351.

Dressler, A., Lynden-Bell, D., Burstein, D., et al. 1987, ApJ, 313, 42

Evstigneeva, E. A., Reshetnikov, V. P., \& Sotnikova, N. Y. 2002, A\&A, 381, 6

Faber, S. M., \& Jackson, R. E. 1976, ApJ, 204, 668

Franx, M., Illingwort, G., \& Heckman, T., 1989, AJ, 98, 2

Franco-Balderas, A., Hernandez-Toledo, H. M., \& Dultzin-Hacyan, D. 2004, A\&A, 417, 411

Galletta, G., Rodighiero, G., Bettoni, D., Moles, M., \& Varela, J. 2006, A\&A, 456, 91

Gonzáles-García, A. C., \& van Albada, T. S. 2003, MNRAS, 342, L36

Gómez, P. L., Nichol, R. C., Miller, C. J., et al. 2003, ApJ, 584, 210

Graham, A. W., \& Driver, S. P. 2005. PASP, 22, 118

Hamabe, M., \& Kormendy, J. 1987, in Structure and Dynamics of Elliptical Galaxies, ed. T. De Zeeuw (Dordrecht: Reidel), IAU Symp., 127, 379

Hashimoto, Y., Oemler, A. J., Lin, H., \& Tucker, D. L. 1998, ApJ, 499, 589

Haynes, M. P., \& Giovanelli, R. 1984, AJ, 89, 758

Hoessel, J. G., Oegerle, W. R., \& Sheider, D. P. 1987, AJ, 94, 1111

Huchra, J., et al. 2000, CfA Redshift Catalog

Icke, V. 1985, A\&A, 144, 115

Isobe, T., Feigenson, E., Akritas, M., \& Babu, G. 1990, ApJ, 364, 104

Jedrzejewski, R. I. 1987, IAUS, 127, 37

Jørgensen, I., \& Franx, M. 1994, ApJ, 433, 553

Jørgensen, I., Franx, M., \& Kærgaard, P. 1992, A\&AS, 95, 489

Jørgensen, I., Franx, M., \& Kærgaard, P. 1993, ApJ, 411, 34

Jørgensen, I., Franx, M., \& Kærgaard, P. 1995, MNRAS, 273, 1097

Jørgensen, I., Franx, M., \& Kærgaard, P. 1996, MNRAS, 280, 167
Jørgensen, I., Franx, M., Hjorth, J., \& van Dokkum, P. G. 1999, MNRAS, 308, 833

Kauffmann, G., \& White, S. D. M. 1993, MNRAS, 261, 921

Kauffmann, G., White, S. D. M., Heckman, T. M., et al. 2004, MNRAS, 341, 54

Kjærgaard, P., Jørgensen, I., Moles, M. 1993, AJ, 418, 617

Kormendy, J. 1985, ApJ, 295, 73

Kuntschner, H., Smith, R. J., Colles, M., et al. 2002, MNRAS, 337, 172

La Barbera, F., Busarello, G., \& Capaccioli, M. 2000, A\&A, 362, 851

La Barbera, F., Busarello, G., Merluzzi, P., \& Massaroti, M. 2003, ApJ, 595, 127

Mahdavi, A., Geller, M. J., Bohringer, H., Kurtz, M. J., \& Ramella, M. 1999, ApJ, 518, 69

Malin, D. F., \& Carter, D. 1983, ApJ, 274, 534

Marcum, P. M., Aars, C. E., \& Fanelli, M. N. 2004, AJ, 127, 3213

Márquez, I., \& Moles, M. 1996, A\&AS, 120, 1

Márquez, I., \& Moles, M. 1999, A\&A, 344, 421

Milvang-Jensen, B. 1997, Master's Thesis, University of Copenhagen

Nieto, J. L., \& Bender, R. 1989, A\&A, 215, 266

Pence, W. ApJ, 1976, 203, 39

Oemler, A. 1974, ApJ, 194, 1

Poggianti, B. M., Smail, I., Dressler, A., et al. 1999, ApJ, 518, 576

Prugniel, P., \& Siemen, F. 1996, A\&A, 309, 749

Prugniel, P., \& Siemen, F. 1997, A\&A, 321, 111

Reda, F. M., Forbes, D. A., Beasley, M., O’Sullivan, E. J., \& Goudfrooij, P. 2004, MNRAS, 354, 851

Reda, F. M., Forbes, D., \& Hau, G. 2005, MNRAS, 360, 693

Reduzzi, L., Longhetti, M., \& Rampazzo, R. 1996, MNRAS, 282, 149

Saglia, R. P., Bertschinger, E., Baggley, G., et al. 1993, MNRAS, 264, 961

Sandage, A., \& Lubin, L. M. 2001, ApJ, 121, 2271

Sandage, A., \& Perelmuter, J. M. 1991, ApJ, 370, 455

Schlegel, D. J., Finkbeiner, D. P., Douglas, P., \& Davis, M. 1998, ApJ, 500, 25

Schweizer, F., Seitzer, P., Faber, S. M., et al. 1990, ApJ, 3641, 33

Seitzer, P., \& Schweizer, F. 1990, Dynamics and Interactions of Galaxies, ed. R. Weilen (New York: Springer), 270

Smith, R. M., Martinez, V. J., \& Graham, M. J. 2003, ApJ, 617, 1017

Stetson, P. B., \& Harris, W. E. 1988, AJ, 96, 909

Stocke, J. T., Keeney, B. A., Lewis, A. D., Epps, H. W., \& Schild, R. E. 2004, AJ, 127, 1336

Tanaka, M., Goto, T., Okamura, S., Shimasaku, K., \& Brinkmann, J. 2004, AJ, 128,2677

Terlevich, M., Davis, R. L., Faber, S. M., \& Burstein, D. 1981, MNRAS, 196, 381

Treu, T., Stiavelli, M., Bertin, G., Casertano, S., \& Møller P. 2001, MNRAS, 326,237

Trujillo, I., Aguerri, J. A. L., Cepa, L., \& Gutiérrez, C. M. 2001a, MNRAS, 321, 269

Trujillo, I., Aguerri, J. A. L., Cepa, L., \& Gutiérrez, C. M. 2001b, MNRAS, 328, 977

Varela, J. 2004, Ph.D. Thesis, Universidad Complutense de Madrid

Varela, J., Moles, M., Márquez, I., et al. 2004, A\&A, 420, 873

White, S. D. M., \& Rees, M. J. 1978, MNRAS, 183, 341

Whitmore, B. C., Gilmore, D. M., \& Jones C. 1993, ApJ, 407, 489

Worthey, G. 1994, ApJS, 95, 107

Ziegler, B. L., Saglia, R. P., Bender, R., et al. 1999, A\&A, 346, 13 
A. Nigoche-Netro et al.: Structural properties of isolated ETG, Online Material $p$ I

\section{Online Material}


A. Nigoche-Netro et al.: Structural properties of isolated ETG, Online Material $p 2$

Table A.1. Catalogue of isolated ETG. Literature data. Column (1) PGC Name. Column (2) other names. Column (3) morphological type from CfA. Column (4) $f$ parameter. Column (5) magnitude in $B$ filter from CfA. Column (6) uncorrected magnitude in $B$ filter from LEDA. Column (7) radial velocity $\left[\mathrm{km} \mathrm{s}^{-1}\right]$ from CfA.

\begin{tabular}{|c|c|c|c|c|c|c|}
\hline$\overline{\text { PGC Name }}$ & Other name & (3) & (4) & $\begin{array}{c}m_{\text {CfA }} \\
B \\
(5)\end{array}$ & $\begin{array}{c}m_{\text {LEDA }} \\
B \\
(6)\end{array}$ & $c z_{\mathrm{CfA}}$ \\
\hline 473 & & -6 & -4.89 & 13.64 & $13.5 \pm 0.3$ & 7730 \\
\hline 718 & & -2 & -5.36 & 14.84 & $14.9 \pm 0.4$ & 6384 \\
\hline 1074 & & -1 & -5.46 & 14.71 & $14.3 \pm 0.3$ & 5240 \\
\hline 1551 & NGC 106 & -5 & -4.81 & 14.37 & $14.5 \pm 0.3$ & 6059 \\
\hline 2170 & & -2 & -4.83 & 16.05 & $15.7 \pm 0.5$ & 9026 \\
\hline 170001 & & -5 & -4.60 & 15.37 & $15.0 \pm 0.4$ & 5868 \\
\hline 2735 & & -2 & -4.54 & 14.87 & $15.1 \pm 0.3$ & 5792 \\
\hline 4606 & IC 0090 & -5 & -4.52 & 14.34 & $14.9 \pm 0.6$ & 5594 \\
\hline 5381 & & -2 & -5.09 & 16.67 & $16.8 \pm 0.1$ & 9686 \\
\hline 5403 & & 0 & -5.74 & 15.39 & $15.1 \pm 0.4$ & 5881 \\
\hline 5655 & & -2 & -4.75 & 15.07 & $14.8 \pm 0.4$ & 5289 \\
\hline 5660 & & -2 & -4.52 & 15.25 & $15.3 \pm 0.2$ & 5546 \\
\hline 6533 & & -2 & -4.82 & 15.29 & $15.3 \pm 0.4$ & 5421 \\
\hline 7658 & & -2 & -4.57 & 14.80 & $14.6 \pm 0.4$ & 5941 \\
\hline 7820 & & 0 & -5.72 & 15.00 & $14.8 \pm 0.3$ & 5513 \\
\hline 8375 & & -2 & -4.59 & 15.5 & $15.5 \pm 0.2$ & 9721 \\
\hline 8617 & & -7 & -4.55 & 15.00 & $14.9 \pm 0.4$ & 8311 \\
\hline 10538 & & 0 & -5.70 & 14.65 & $14.0 \pm 0.4$ & 7618 \\
\hline 10613 & NGC 1101 & -2 & -5.67 & 13.96 & $13.9 \pm 0.1$ & 7093 \\
\hline 10947 & & -4 & -4.55 & 14.55 & $14.7 \pm 0.4$ & 5362 \\
\hline 11159 & & -4 & -4.58 & 15.02 & $15.1 \pm 0.4$ & 7686 \\
\hline 11254 & & -2 & -5.16 & 15.21 & $15.2 \pm 0.1$ & 6333 \\
\hline 11749 & NGC 1218 & 0 & -4.75 & 13.96 & $13.9 \pm 0.1$ & 8647 \\
\hline 13088 & NGC 1349 & -2 & -5.68 & 14.17 & $14.2 \pm 0.2$ & 6594 \\
\hline 170053 & & -2 & -5.03 & 14.95 & $14.9 \pm 0.4$ & 8448 \\
\hline 14141 & & -2 & -4.76 & 15.50 & $15.5 \pm 0.2$ & 6365 \\
\hline 170059 & & -2 & -5.01 & 15.65 & $15.6 \pm 0.3$ & 6869 \\
\hline 14810 & & -2 & -4.51 & 15.64 & $15.6 \pm 0.2$ & 9908 \\
\hline 27702 & & -1 & -4.86 & 15.66 & $15.3 \pm 0.4$ & 8543 \\
\hline 27931 & IC 0558 & -3 & -4.58 & 15.23 & $14.9 \pm 0.4$ & 9334 \\
\hline 28009 & & -2 & -4.83 & 14.58 & $14.3 \pm 0.6$ & 5020 \\
\hline 31768 & NGC 3332 & -2 & -5.29 & 14.01 & $13.6 \pm 0.6$ & 5867 \\
\hline 31920 & & -1 & -5.37 & 15.47 & $15.2 \pm 0.4$ & 6093 \\
\hline 32231 & NGC 3376 & 0 & -5.22 & 14.57 & $13.9 \pm 0.4$ & 5837 \\
\hline 32672 & & -6 & -5.08 & 14.68 & & 5793 \\
\hline 33460 & & -1 & -4.98 & 14.12 & $13.9 \pm 0.4$ & 6303 \\
\hline 33662 & IC 0669 & 0 & -4.63 & 14.84 & $14.3 \pm 0.4$ & 8786 \\
\hline 33917 & & -1 & -4.52 & 16.60 & $15.7 \pm 0.8$ & 7607 \\
\hline 34159 & NGC 3580 & -1 & -4.68 & 15.51 & $14.8 \pm 0.5$ & 6788 \\
\hline 165572 & & -2 & -5.42 & 15.35 & $15.5 \pm 0.3$ & 7455 \\
\hline 170134 & & -5 & -4.70 & 15.35 & $15.3 \pm 0.3$ & 7191 \\
\hline 35545 & NGC 3716 & -1 & -4.52 & 14.42 & $14.3 \pm 0.4$ & 6628 \\
\hline 36658 & IC 0730 & 0 & -4.62 & 15.40 & $14.9 \pm 0.5$ & 6065 \\
\hline 36946 & & -5 & -5.23 & 15.44 & $15.1 \pm 0.5$ & 5629 \\
\hline 37105 & & -2 & -4.55 & 15.14 & $14.9 \pm 0.4$ & 6432 \\
\hline 38043 & & -2 & -4.55 & 15.90 & $15.6 \pm 0.4$ & 9633 \\
\hline 38195 & & -2 & -4.69 & 15.31 & $14.8 \pm 0.6$ & 6736 \\
\hline 39120 & NGC 4201 & -2 & -4.75 & 14.67 & $14.5 \pm 0.3$ & 5406 \\
\hline 42367 & IC 0803 & -5 & -4.86 & 15.35 & 15.5. \pm 0.5 & 7985 \\
\hline 42470 & & -2 & -4.69 & 15.30 & $14.9 \pm 0.2$ & 8524 \\
\hline 42721 & & -5 & -4.74 & 15.43 & $15.4 \pm 0.3$ & 6600 \\
\hline 43143 & NGC 4685 & -3 & -4.85 & 13.22 & $13.6 \pm 0.3$ & 6760 \\
\hline 43848 & IC 0832 & -4 & -4.62 & 15.15 & $15.1 \pm 0.4$ & 7094 \\
\hline 44175 & & 0 & -5.20 & 14.74 & $14.9 \pm 0.4$ & 5500 \\
\hline 83703 & & -4 & -4.59 & 16.22 & & 7339 \\
\hline 170226 & & -2 & -4.57 & 15.14 & $15.6 \pm 0.5$ & 5924 \\
\hline 45821 & & -2 & -5.01 & 15.63 & $15.4 \pm 0.4$ & 6793 \\
\hline
\end{tabular}


A. Nigoche-Netro et al.: Structural properties of isolated ETG, Online Material p 3

Table A.1. continued.

\begin{tabular}{ccccccc}
\hline \hline PGC Name & Other name & $t$ & $f$ & $m_{\text {CfA }}$ & $m_{\text {LEDA }}$ & $c z_{\text {CfA }}$ \\
& & & & $B$ & $B$ & $(7)$ \\
\hline 158586 & $(2)$ & $(3)$ & $(4)$ & $(5)$ & $(6)$ & $(7)$ \\
48635 & IC 4322 & -4 & -5.20 & 15.20 & $15.1 \pm 0.4$ & 5372 \\
49487 & & -2 & -4.80 & 14.86 & $14.9 \pm 0.4$ & 7630 \\
51313 & NGC 5583 & -5 & -5.54 & 14.20 & $13.9 \pm 0.4$ & 5007 \\
51699 & NGC 5628 & -7 & -5.30 & 14.77 & $14.5 \pm 0.4$ & 5724 \\
52601 & & -3 & -5.86 & 15.21 & $14.9 \pm 0.3$ & 7019 \\
52667 & IC 4498 & -2 & -4.67 & 15.06 & $14.9 \pm 0.3$ & 9309 \\
53404 & & -2 & -4.61 & 14.55 & $14.8 \pm 0.3$ & 7691 \\
53449 & & -3 & -5.64 & 15.76 & $15.5 \pm 0.4$ & 9381 \\
55684 & NGC 5972 & 0 & -5.00 & 15.19 & $14.8 \pm 0.4$ & 8959 \\
170386 & & -2 & -5.42 & 14.90 & $14.8 \pm 0.3$ & 5261 \\
68438 & IC 1437 & 0 & -4.53 & 14.44 & $14.6 \pm 0.3$ & 8911 \\
68934 & & -2 & -4.54 & 15.49 & $15.4 \pm 0.2$ & 7529 \\
133427 & & -2 & -4.71 & 15.28 & $15.5 \pm 0.2$ & 8276 \\
69548 & & -2 & -4.80 & 15.26 & $15.5 \pm 0.3$ & 7262 \\
69782 & & -4 & -6.00 & 14.94 & $14.8 \pm 0.38$ & 8567 \\
70086 & IC 1460 & -5 & -4.96 & 15.72 & $15.0 \pm 0.1$ & 7255 \\
70499 & & -2 & -4.59 & 14.76 & $14.9 \pm 0.4$ & 7141 \\
A2313-0514 & & -2 & -4.70 & 15.60 & & 7202 \\
71127 & & -3 & -5.89 & 15.40 & $15.3 \pm 0.5$ & 5680 \\
71203 & & -2 & -4.89 & 14.95 & $15.2 \pm 0.4$ & 5960 \\
71254 & & -1 & -4.55 & 15.44 & $15.4 \pm 0.4$ & 9246 \\
71712 & IC 7692 & -5 & -5.20 & 16.01 & $15.2 \pm 0.6$ & 5247 \\
72565 & & -2 & -4.72 & 15.25 & $15.3 \pm 0.5$ & 5556 \\
16 & & -2 & -4.55 & 15.05 & $15.1 \pm 0.3$ & 5616 \\
\hline
\end{tabular}


A. Nigoche-Netro et al.: Structural properties of isolated ETG, Online Material p 4

Table A.5. Catalogue of perturbed S0 galaxies in $B$ filter. Column (1) NGC or IC Name. Column (2) mean surface brightness [mag arcsec ${ }^{-2}$ ] within effective radius in $B$ filter. Column (3) logarithm of effective radius $\left[r_{\mathrm{e}}\right.$ in kpc] in $B$ filter. Column (4) total apparent magnitude in $B$ filter. Column (5) total absolute magnitude in $B$ filter. Column (6) CMB velocity $\left[\mathrm{km} \mathrm{s}^{-1}\right]$. Column (7) environment information. Column (8) references of environment information.

\begin{tabular}{cccccccc}
\hline \hline $\begin{array}{c}\text { NGC or IC Name } \\
(1)\end{array}$ & $\begin{array}{c}\left\langle S B_{\mathrm{e}}\right\rangle \\
(2)\end{array}$ & $\begin{array}{c}\log \left(r_{\mathrm{e}}\right) \\
(3)\end{array}$ & $\begin{array}{c}m_{T} \\
(4)\end{array}$ & $\begin{array}{c}M_{T} \\
(5)\end{array}$ & $\begin{array}{c}c z_{\mathrm{CMB}} \\
(6)\end{array}$ & $\begin{array}{c}\text { Environment information } \\
(7)\end{array}$ & $\begin{array}{c}\text { References of enviroment information } \\
(8)\end{array}$ \\
\hline 474 & 21.47 & 0.58 & 12.50 & -19.99 & 2360 & Group & Karachentseva (1979) \\
2911 & 23.21 & 1.10 & 12.26 & -20.87 & 3172 & Group & Denicolo et al. (2005) \\
3384 & 19.83 & 0.09 & 10.85 & -19.17 & 755 & Cluster & group \\
3414 & 20.61 & 0.34 & 11.87 & -19.65 & 1508 & Group Bergh $(2002)$ \\
3801 & 22.44 & 0.81 & 13.10 & -20.17 & 3379 & Cluster & Ramella et al. (2005) \\
3998 & 20.00 & 0.21 & 11.48 & -19.64 & 1256 & Cluster & van den Bergh (2002) \\
4350 & 20.38 & 0.23 & 11.85 & -19.35 & 1304 & Cluster & Binggeli et al. (1985) \\
4382 & 21.11 & 0.56 & 09.87 & -20.27 & 800 & Cluster & Binggeli et al. (1985) \\
4638 & 20.15 & 0.12 & 11.99 & -19.00 & 1183 & Cluster & Binggeli et al. (1985) \\
5308 & 20.98 & 0.49 & 12.19 & -20.08 & 2128 & Cluster & van den Bergh (2002) \\
535 van den Bergh $(2002)$ \\
763
\end{tabular}


A. Nigoche-Netro et al.: Structural properties of isolated ETG, Online Material p 5

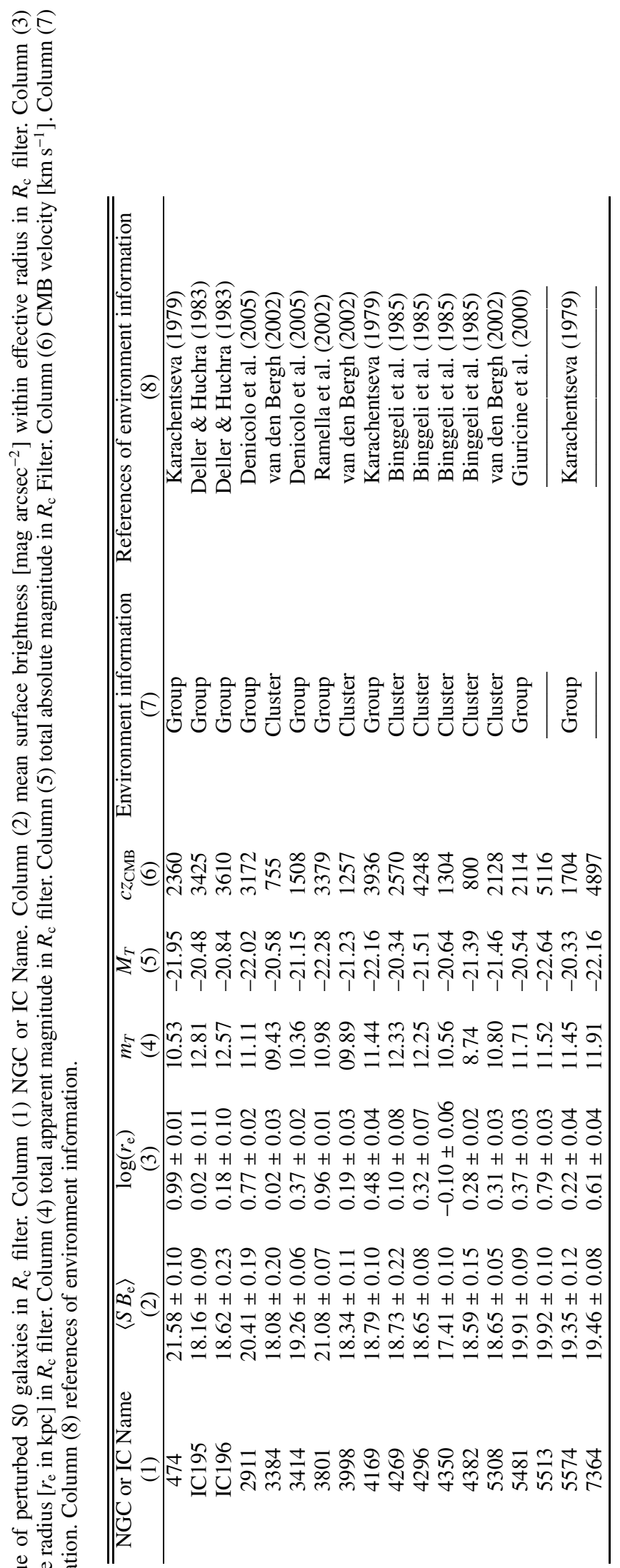


A. Nigoche-Netro et al.: Structural properties of isolated ETG, Online Material $p 6$
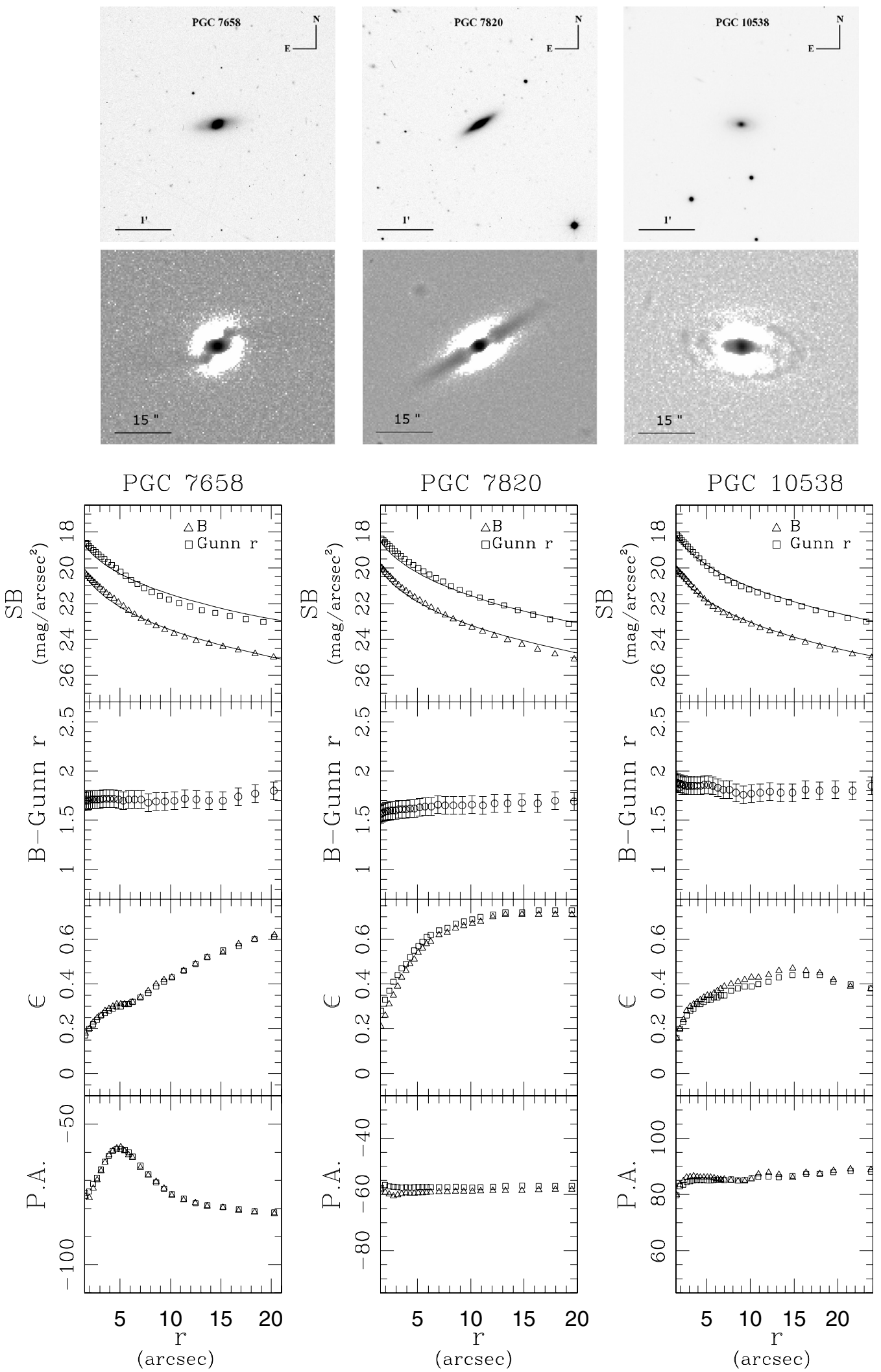

Fig. B.2. Each panel contains: image in $B$ filter, filtered image in $B$ filter, $B$ and Gunn $r$ filter surface brightness (the continuous line represents the de Vaucouleurs fit), $B$ - Gunn $r$ colour, ellipticity $(\epsilon)$ profile and position angle (PA) profile. Both the surface brightness profile and the colour profile are shown with no corrections. The $B$ and Gunn $r$ filter data are represented by squares and triangles respectively. 
A. Nigoche-Netro et al.: Structural properties of isolated ETG, Online Material $p 7$
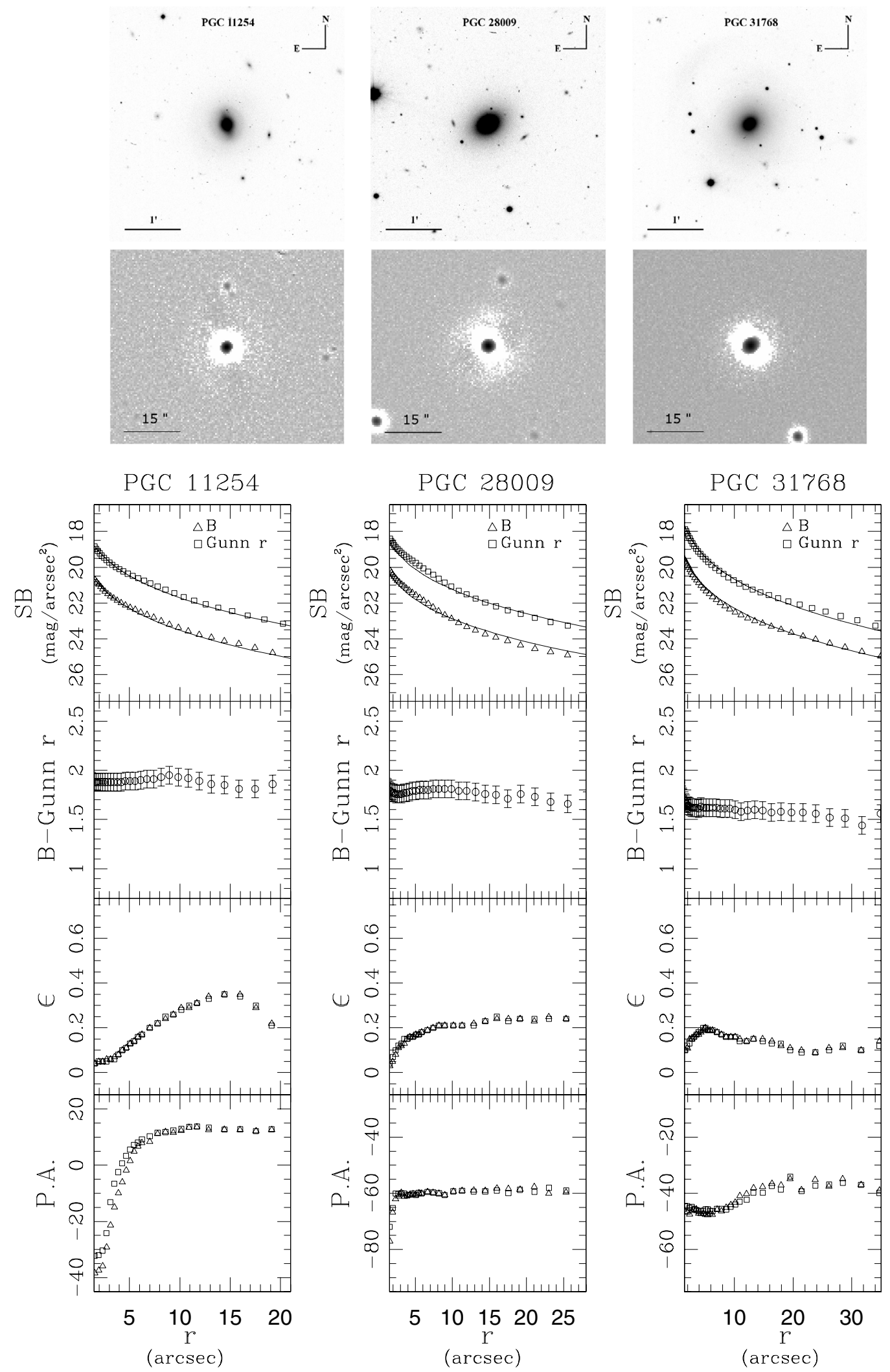

Fig. B.3. Each panel contains: image in $B$ filter, filtered image in $B$ filter, $B$ and Gunn $r$ filter surface brightness (the continuous line represents the de Vaucouleurs fit), $B$ - Gunn $r$ colour, ellipticity $(\epsilon)$ profile and position angle (PA) profile. Both the surface brightness profile and the colour profile are shown with no corrections. The $B$ and Gunn $r$ filter data are represented by squares and triangles respectively. 
A. Nigoche-Netro et al.: Structural properties of isolated ETG, Online Material $p 8$
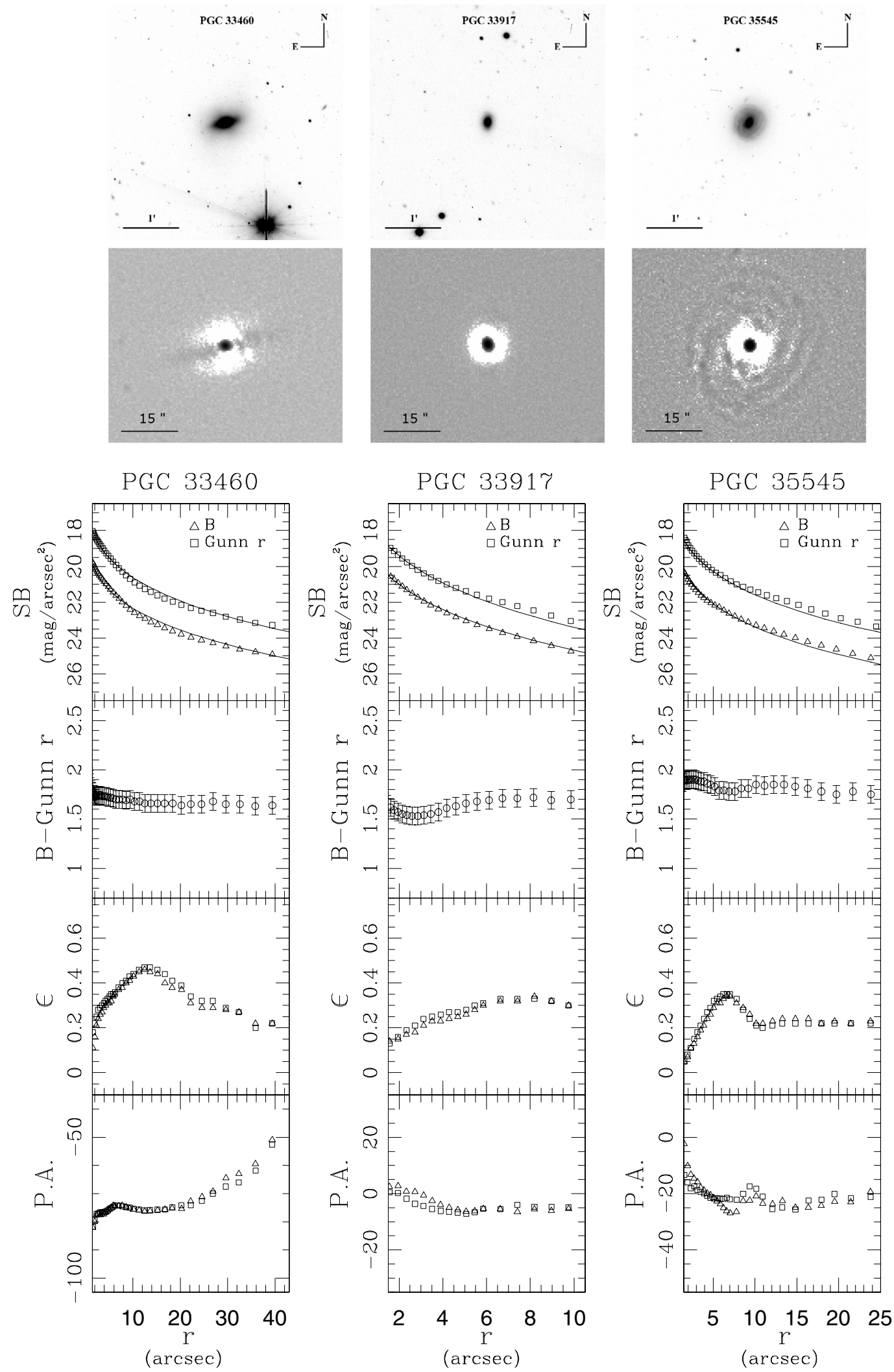

Fig. B.4. Each panel contains: image in $B$ filter, filtered image in $B$ filter, $B$ and Gunn $r$ filter surface brightness (the continuous line represents the de Vaucouleurs fit), $B$ - Gunn $r$ colour, ellipticity $(\epsilon)$ profile and position angle (PA) profile. Both the surface brightness profile and the colour profile are shown with no corrections. The $B$ and Gunn $r$ filter data are represented by squares and triangles respectively. 
A. Nigoche-Netro et al.: Structural properties of isolated ETG, Online Material $p 9$
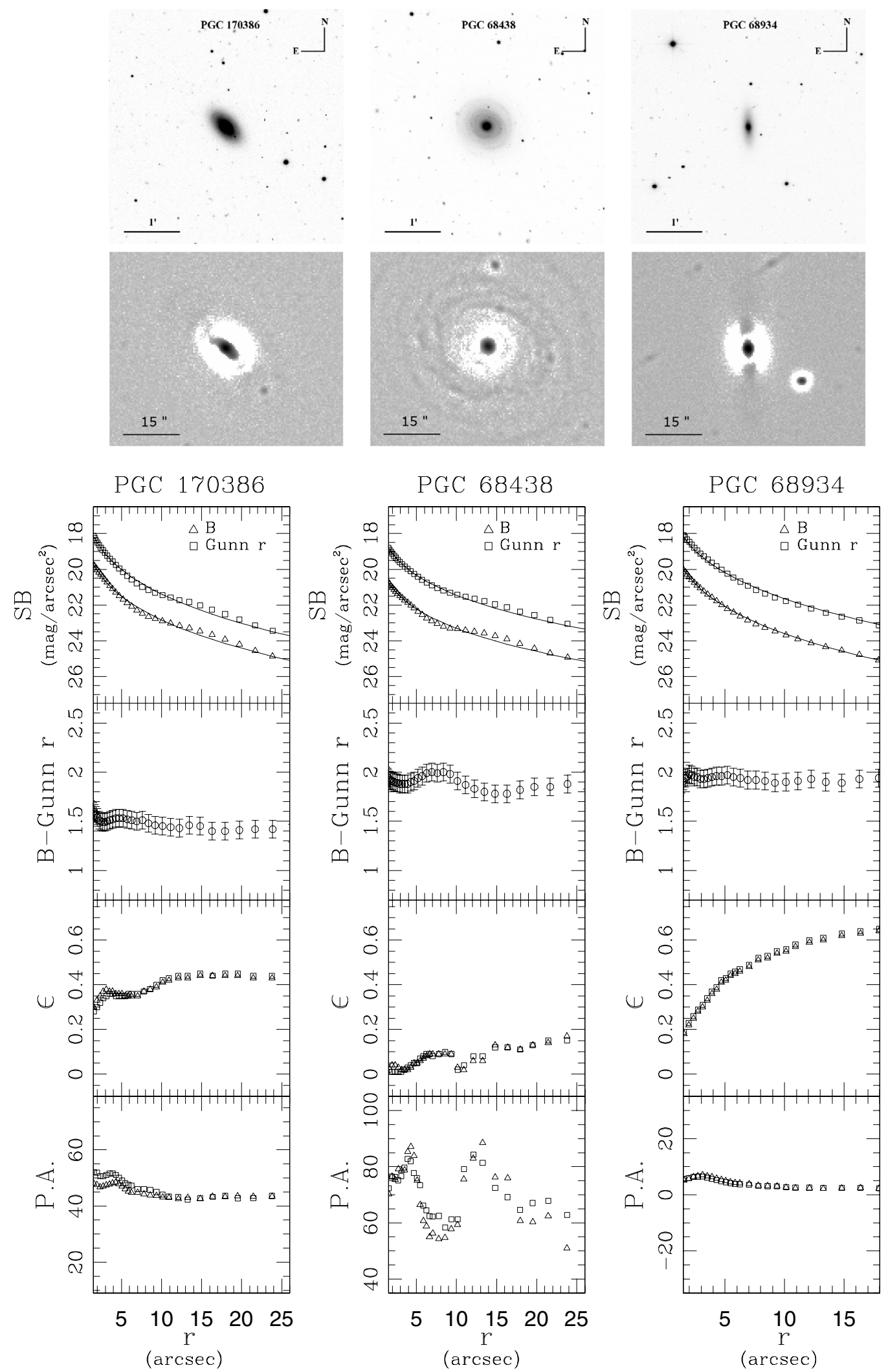

Fig. B.5. Each panel contains: image in $B$ filter, filtered image in $B$ filter, $B$ and Gunn $r$ filter surface brightness (the continuous line represents the de Vaucouleurs fit), $B$ - Gunn $r$ colour, ellipticity $(\epsilon)$ profile and position angle (PA) profile. Both the surface brightness profile and the colour profile are shown with no corrections. The $B$ and Gunn $r$ filter data are represented by squares and triangles respectively. 
A. Nigoche-Netro et al.: Structural properties of isolated ETG, Online Material $p 10$
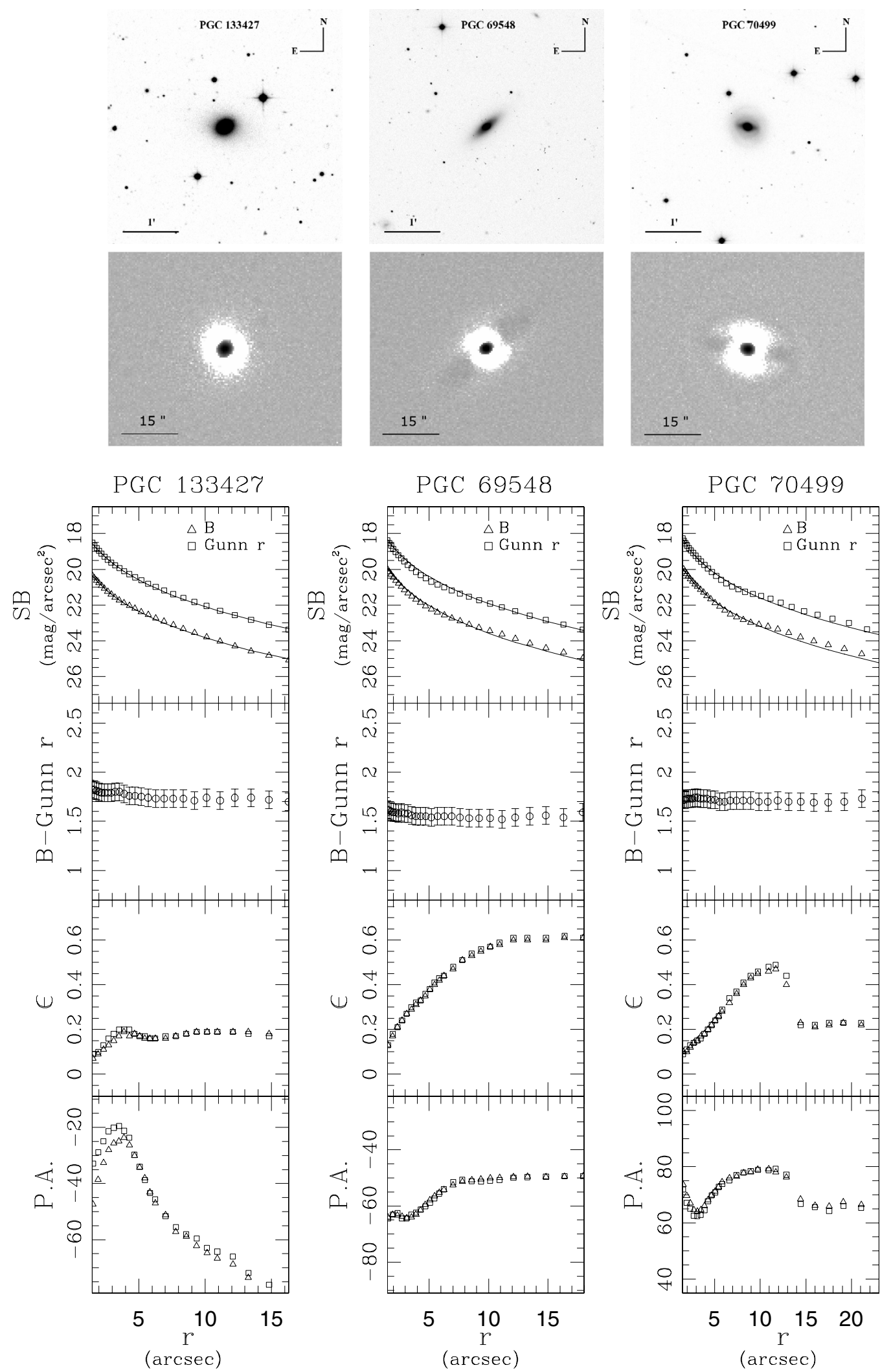

Fig. B.6. Each panel contains: image in $B$ filter, filtered image in $B$ filter, $B$ and Gunn $r$ filter surface brightness (the continuous line represents the de Vaucouleurs fit), $B$ - Gunn $r$ colour, ellipticity $(\epsilon)$ profile and position angle (PA) profile. Both the surface brightness profile and the colour profile are shown with no corrections. The $B$ and Gunn $r$ filter data are represented by squares and triangles respectively. 
A. Nigoche-Netro et al.: Structural properties of isolated ETG, Online Material $p 11$
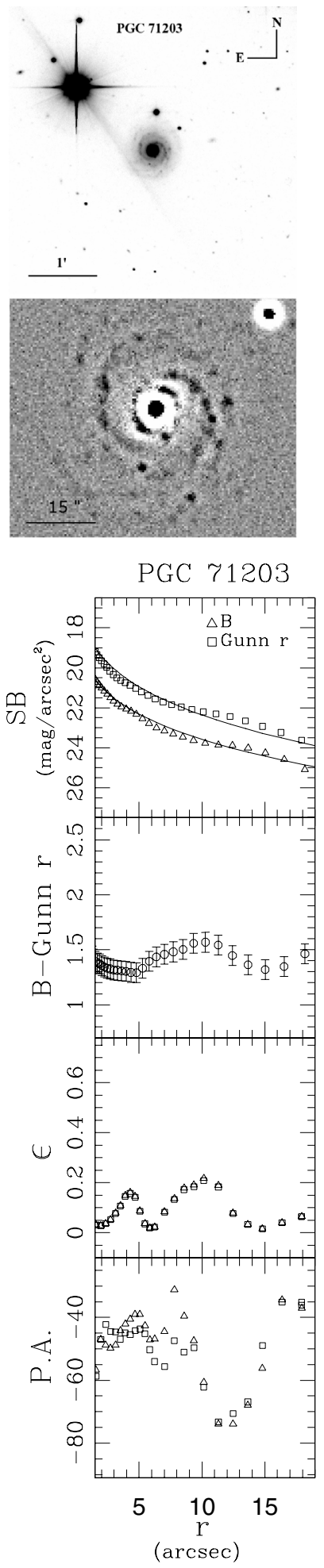

Fig. B.7. Each panel contains: image in $B$ filter, filtered image in $B$ filter, $B$ and Gunn $r$ filter surface brightness (the continuous line represents the de Vaucouleurs fit), $B-$ Gunn $r$ colour, ellipticity $(\epsilon)$ profile and position angle (PA) profile. Both the surface brightness profile and the colour profile are shown with no corrections. The $B$ and Gunn $r$ filter data are represented by squares and triangles respectively. 
A. Nigoche-Netro et al.: Structural properties of isolated ETG, Online Material $p 12$
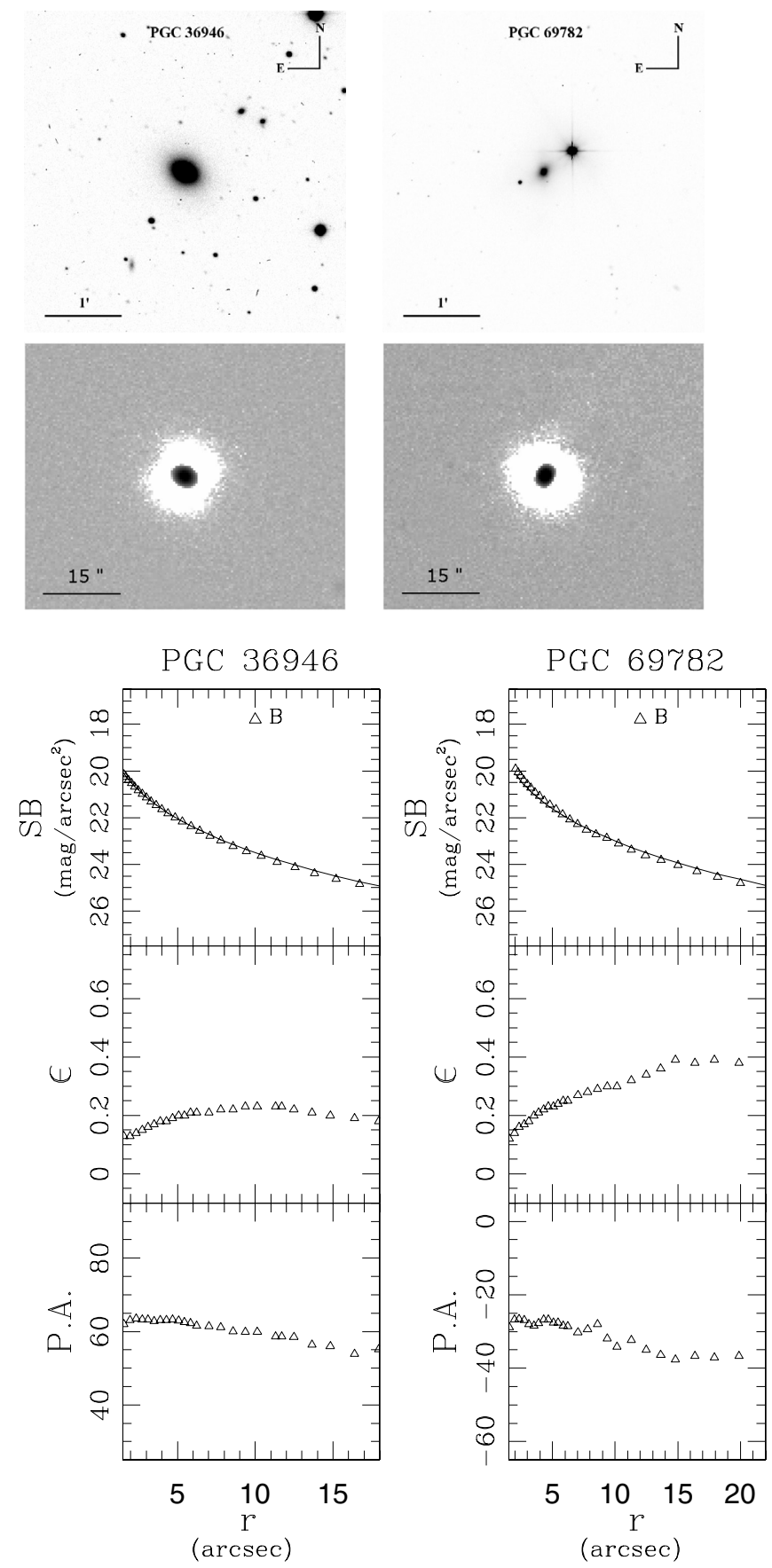

Fig. B.8. Each panel contains: image in $B$ filter, filtered image in $B$ filter, $B$ filter surface brightness (the continuous line represents the de Vaucouleurs fit), ellipticity $(\epsilon)$ profile and position angle (PA) profile. The surface brightness profile are shown with no corrections. 
A. Nigoche-Netro et al.: Structural properties of isolated ETG, Online Material $p 13$
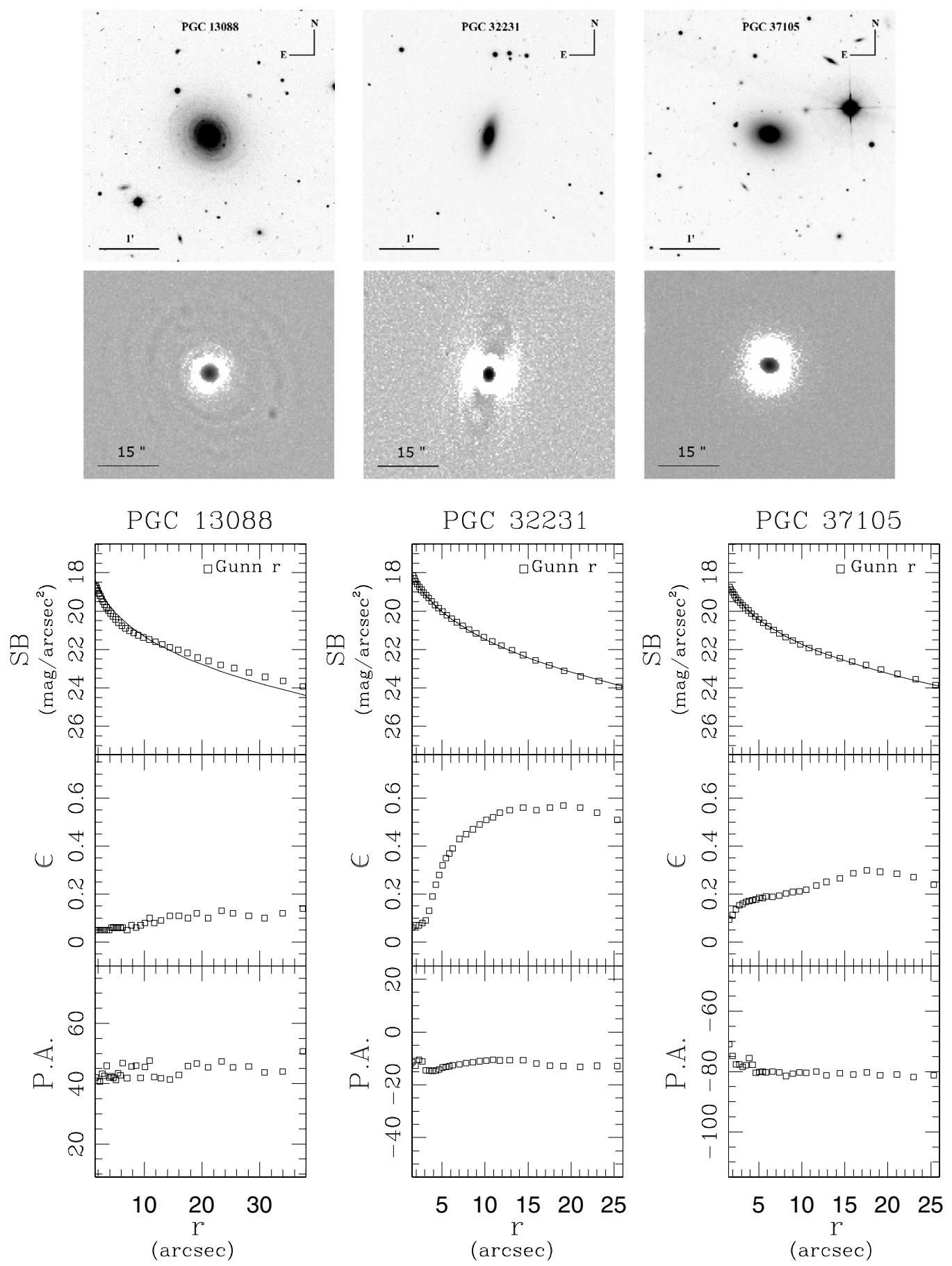

Fig. B.9. Each panel contains: image in Gunn $r$ filter, filtered image in Gunn $r$ filter, Gunn $r$ filter surface brightness (the continuous line represents the de Vaucouleurs fit), ellipticity $(\epsilon)$ profile and position angle (PA) profile. The surface brightness profile are shown with no corrections. 